\title{
Study of single sneutrino production in association with fermion pairs at polarized photon colliders
}

\author{
Dilip Kumar Ghosh* \\ Department of Physics, National Taiwan University, Taipei, Taiwan 10617, Republic of China
}

Stefano Moretti ${ }^{\dagger}$

CERN Theory Division, CH-1211 Geneva 23, Switzerland

and Institute for Particle Physics Phenomenology, University of Durham, Durham DH1 3LE, United Kingdom

(Received 25 January 2002; revised manuscript received 28 May 2002; published 23 August 2002)

\begin{abstract}
We investigate single sneutrino production in the context of $R$-parity-violating supersymmetry at future $\gamma \gamma$ linear colliders. The sneutrino is produced in association with fermion pairs and it is shown that its decays into two further fermions will lead to a clean signal. We also discuss possible standard model backgrounds and the effects of beam polarization.
\end{abstract}

DOI: 10.1103/PhysRevD.66.035004

PACS number(s): 12.60.Jv, 13.10.+q, 14.80.Ly

\section{INTRODUCTION}

Supersymmetry (SUSY) is currently the most attractive theoretical framework describing physics beyond the standard model (SM). Even the minimal extension of the SM incorporating SUSY (MSSM) predicts a zoo of new particles, which have not yet been observed. One of the major areas of activity in high energy physics today and in the near future is to prove their existence. If SUSY is realized at the electroweak (EW) scale, many of the superparticles should be discovered at the next generation of hadron colliders, such as the Tevatron (run II, $\sqrt{s_{p \bar{p}}}=2 \mathrm{TeV}$ ) at Fermilab and the Large Hadron Collider (LHC, $\sqrt{s_{p p}}=14 \mathrm{TeV}$ ) at CERN. These machines, while having the chance of being the first to access the SUSY domain, are, however, hampered by the fact that a large QCD background and the lack of knowledge of the initial center-of-mass (CM) partonic energies render the task of determining sparticle properties (masses, couplings, quantum numbers, etc.) difficult. Insight into this "SUSY spectrum" would in fact shed light on the yet unknown mechanism leading to SUSY breaking.

In contrast, in $e^{+} e^{-}$collisions, the QCD noise is under control and the initial energies of the leptons are generally well known. This has contributed in recent years to the generation of a strong consensus behind the option of building electron-positron linear colliders (LCs), operating in the energy range from $500 \mathrm{GeV}$ to $3 \mathrm{TeV}$, as the accelerators most suited to inherit the legacy of the run II and LHC era [1]. Such machines would not only provide the ideal environment for discovering the SUSY particles which could be missed at the Fermilab and CERN experiments, but would also allow for the precise determination of the mentioned SUSY spectrum. For example, mass measurements are aided by the ability to perform threshold scans by varying the collider c.m. energy. Furthermore, the spin properties of many SUSY particles can be accessed by exploiting an efficient beam polarization, a feature altogether missing at the Tevatron and the LHC.

\footnotetext{
*Email address: dghosh@phys.ntu.edu.tw

†Email address: stefano.moretti@cern.ch
}

Another advantage of LCs is that they can easily be converted to run quite simply in the $e^{-} e^{-}$mode or even in $e \gamma$ and $\gamma \gamma$, the latter by using Compton backscattering of laser photons against the electrons or positrons [2,3], all such collisions taking place with energy and luminosity comparable to those obtainable from the primary $e^{+} e^{-}$design. Quite apart from SUSY [4], it should be recalled that electronelectron collisions would constitute a privileged window on, e.g., models with extended Higgs sectors, whereas those employing photon beams would easily allow for, e.g., the study of a plethora of QCD topics.

To come back to SUSY, it should be mentioned that there have been in the recent years quite promising explorations of the physics potential of $\gamma \gamma$ LCs as a probe of the low energy dynamics of the theory [5]. It is the intention of our study to further dwell on this topic by considering the scope of LCs in accessing some $R$-parity-violating (RPV) signals of SUSY.

\section{II. $R$-PARITY-VIOLATING SUPERSYMMETRY}

The construction of the most general Supersymmetric extension of the SM leads to baryon- (B-) and lepton- (L-) number-violating operators in the superpotential

$$
W_{k}=\lambda_{i j k} \hat{L}_{i} \hat{L}_{j} \hat{E}_{k}^{c}+\lambda_{i j k}^{\prime} \hat{L}_{i} \hat{Q}_{j} \hat{D}_{k}^{c}+\epsilon_{i} \hat{L}_{i} \hat{H}_{2}+\lambda_{i j k}^{\prime \prime} \hat{U}_{i}^{c} \hat{D}_{j}^{c} \hat{D}_{k}^{c} .
$$

Here, $\hat{H}_{1}, \hat{H}_{2}$ are the $S U(2)$ doublets Higgs superfields which give rise to the masses of down-type and up-type quark superfields, respectively, $\hat{L}(\hat{Q})$ denotes lepton(quark) doublet superfields, $\hat{E}^{c}, \hat{D}^{c}, \hat{U}^{c}$ are the singlet lepton and quark superfields, $i, j, k$ are the generational indices and we have suppressed the $S U(2)$ and $S U(3)$ indices. The $\lambda_{i j k}$ are antisymmetric in $i$ and $j$ while the $\lambda_{i j k}^{\prime \prime}$ are antisymmetric in $j$ and $k$. The first three terms in $W_{k}$ violate lepton number and the last term violates baryon number conservation. The simultaneous presence of both B- and L-violating operators would induce rapid proton decay which would contradict the strict experimental bound of [6]. In order to keep the proton lifetime within the experimental limit, one needs to impose an additional symmetry beyond the SM gauge symmetry, in 

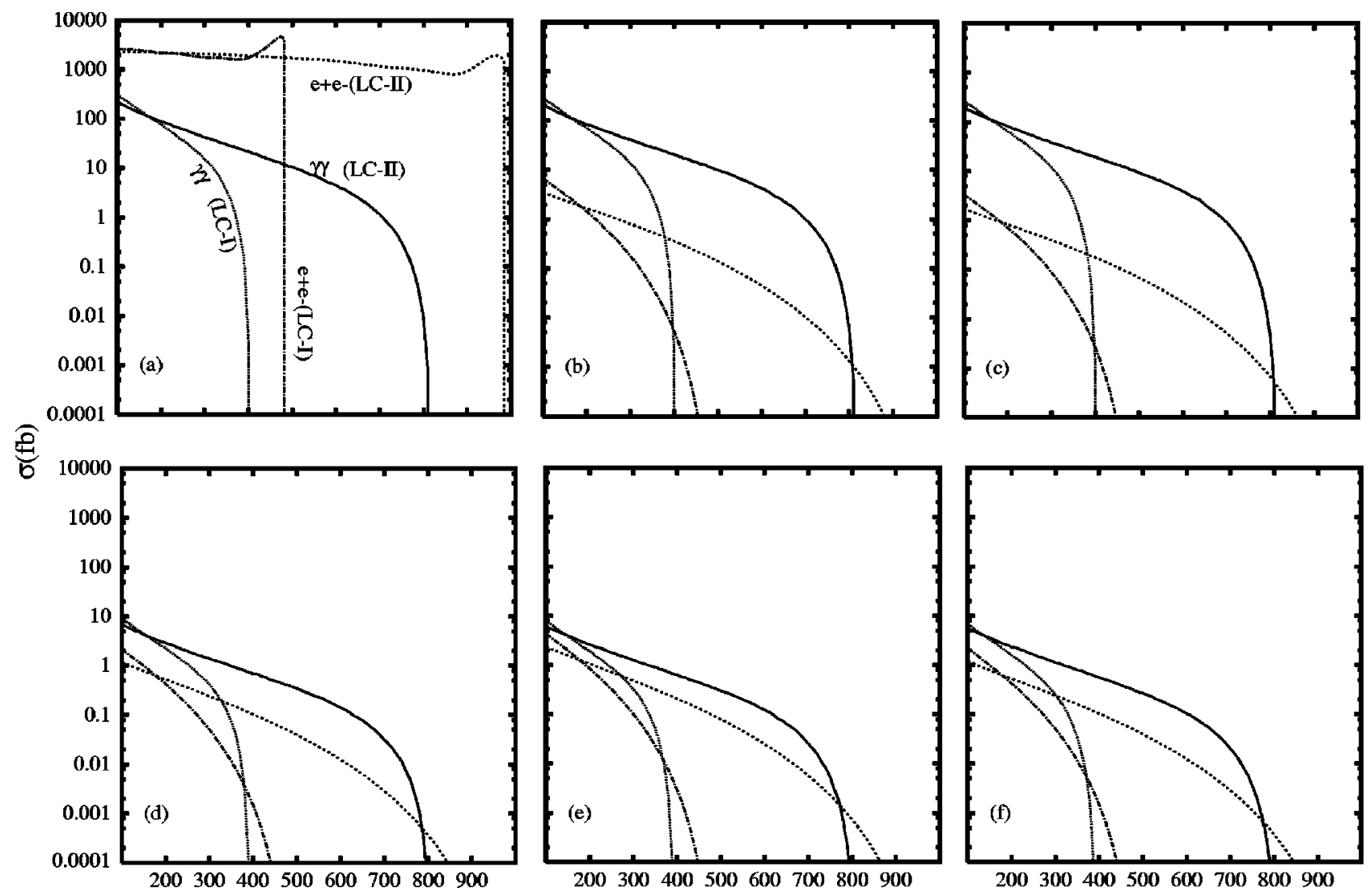

Sneutrino Mass (GeV)

FIG. 1. Cross sections for the following production processes: (a) $\tilde{\nu} e^{+} e^{-}$, (b) $\tilde{\nu} \tau^{+} \mu^{-}+$c.c., (c) $\tilde{\nu} \tau^{+} \tau^{-}$, (d) $\tilde{\nu} s \bar{s}$, (e) $\tilde{\nu} b \bar{s}+$ c.c., and (f) $\tilde{\nu} b \bar{b}$, at a $\gamma \gamma$ (solid) and $e^{+} e^{-}$(dashed) collider, as a function of the sneutrino mass $M_{\tilde{\nu}}$, at $\sqrt{s_{e^{+} e^{-}}}=500 \mathrm{GeV}$ (LC-I) and $1 \mathrm{TeV}$ (LC-II), after the cuts in Eq. (10). For simplicity, we have set the parity violating couplings $\lambda$ and $\lambda^{\prime}$ to 1 .

order to force the unwanted B- and L-violating interactions to vanish. In most cases this can be achieved by imposing a discrete symmetry, called $R$ parity [7], defined as $R=$ $(-1)^{3 \mathrm{~B}+\mathrm{L}+2 \mathrm{~S}}$, where $\mathrm{S}$ is the spin. This symmetry not only forbids rapid proton decay [8] but also renders stable the lightest supersymmetric particle (LSP).

However, $R$ parity is quite an ad hoc assumption in nature, as there are no strong theoretical arguments to support it. Therefore it is much justified to investigate the phenomenological consequences of RPV SUSY. Extensive studies have been carried out in order to look for direct as well as indirect evidence of trilinear $R$-parity violation in different processes at various colliders as well as in order to put constraints on various RPV couplings [9].

Resonant sneutrino production in $\gamma \gamma$ collisions has been studied in Ref. [12], where the rare decays into two photons or gluons were considered. In this paper we will consider instead RPV single production of sneutrinos in association with fermion pairs in polarized photon-photon collisions at $500 \mathrm{GeV}$ and $1 \mathrm{TeV} \mathrm{LCs}$, and their subsequent decays into two further fermions, via trilinear L-violating operators, while preserving B-conservation. The latter channel is in our opinion more suited as a sneutrino "search" mode in $\gamma \gamma$ collisions than the former, simply because one can scan a wider range of sneutrino masses $M_{\tilde{\nu}}$ (as long as $\sqrt{s_{\gamma \gamma}}$ $\approx 0.8 \sqrt{s_{e^{+} e^{-}}} \gg M \tilde{\nu}$ ), thanks to the fact that some amount of energy is carried away by the accompanying fermion pair, whereas in direct production the only $M_{\tilde{\nu}}$ attainable is basically the (reduced) c.m. energy itself. Furthermore, the associate mode may also induce flavor changing final states, so that, as pointed out in [13], unlike in the case of resonant production, one has that the corresponding signatures are basically SM background free. Schematically, one has

TABLE I. Experimental $(2 \sigma)$ upper bounds on the RPV couplings relevant to this analysis. All sfermion masses are assumed to be $100 \mathrm{GeV}$.

\begin{tabular}{lcc}
\hline \hline Coupling & Upper Limit & Sources \\
$\lambda_{311}$ & 0.062 & $R_{\tau}=\frac{\Gamma(\tau \rightarrow e \nu \bar{\nu})}{\Gamma(\tau \rightarrow e \mu \bar{\nu})}[11]$ \\
$\lambda_{323}$ & 0.070 & $R_{\tau}=\frac{\Gamma(\tau \rightarrow e \nu \bar{\nu})}{\Gamma(\tau \rightarrow e \mu \bar{\nu})}[11]$ \\
$\lambda_{323}^{\prime}$ & 0.52 & $R_{D_{s}}=\frac{\Gamma\left(D_{s} \rightarrow \tau \nu_{\tau}\right)}{\Gamma\left(D_{s} \rightarrow \mu \nu_{\mu}\right)}[23]$ \\
$\lambda_{333}^{\prime}$ & 0.45 & $R_{D_{s}}=\frac{\Gamma\left(D_{s} \rightarrow \tau \nu_{\tau}\right)}{\Gamma\left(D_{s} \rightarrow \mu \nu_{\mu}\right)}[23]$ \\
\hline \hline
\end{tabular}



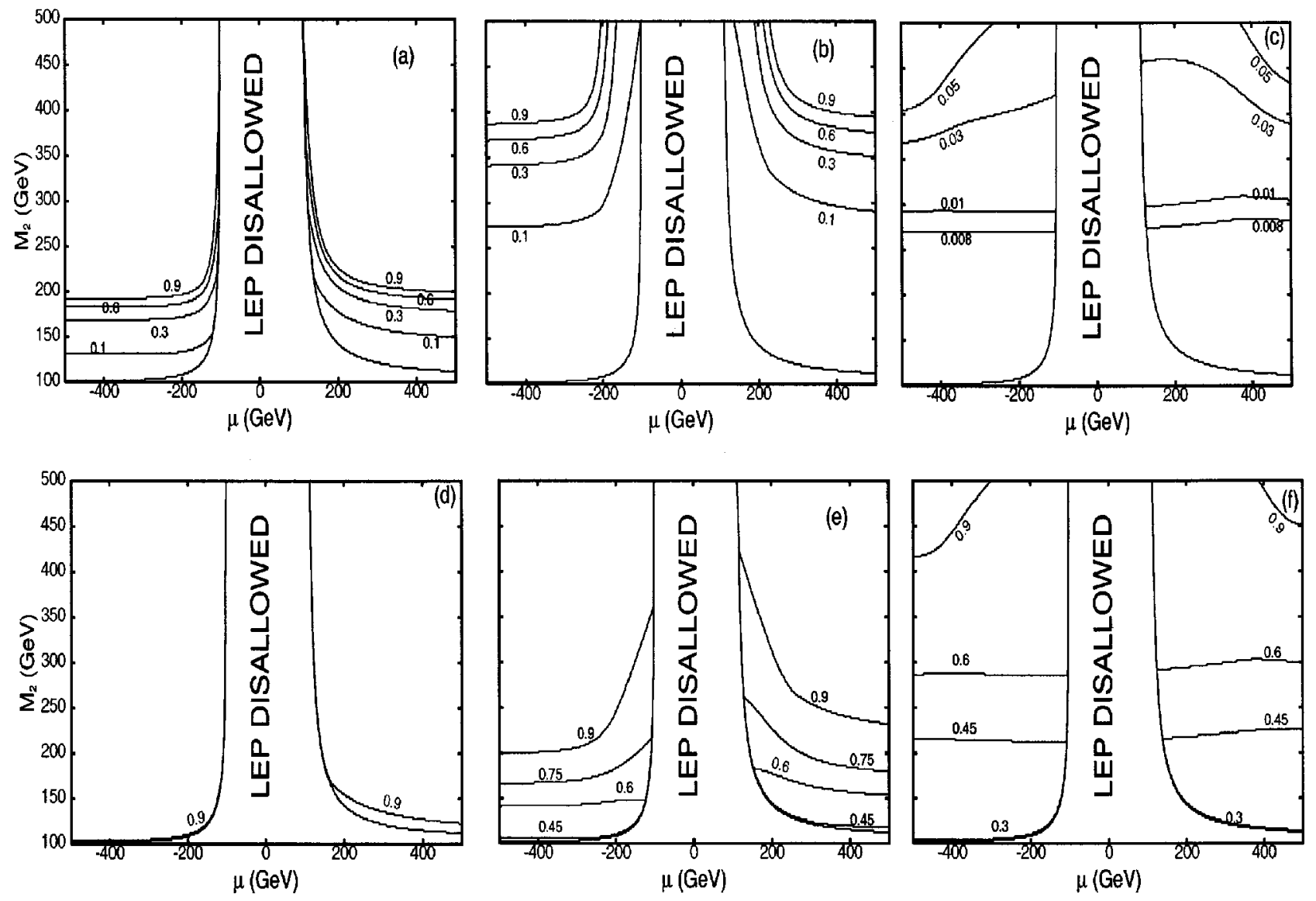

FIG. 2. Constant BR contours of the decay $\tilde{\nu}_{\tau} \rightarrow e^{+} e^{-}$for three values of sneutrino masses: $100 \mathrm{GeV}$ (a), $200 \mathrm{GeV}$ (b), and $400 \mathrm{GeV}$ (c). (d)-(f) represent contours of constant $\mathrm{BR}\left(\tilde{\nu}_{\tau} \rightarrow b \bar{b}\right)$, again for a 100, 200, and $400 \mathrm{GeV}$ sneutrino mass, respectively over $\mu-M_{2}$ plane. The relevant L-violating couplings are here: $\lambda_{311}=0.062$ for (a) $-(\mathrm{c})$ and $\lambda_{333}^{\prime}=0.45$ for (d) $-(\mathrm{f})$. The other relevant MSSM parameters are those of set $\mathrm{A}$ in Table II below.

$$
\gamma \gamma \rightarrow \tilde{\nu} l^{ \pm} l^{\prime} \mp \quad \text { or } \quad \tilde{\nu} q \bar{q}^{\prime}
$$

with

$$
\widetilde{\nu} \rightarrow l^{\prime \prime} l^{\prime \prime \prime} \mp \quad \text { or } \quad \tilde{\nu} \rightarrow q^{\prime \prime} \bar{q}^{\prime \prime \prime}
$$

where the l's refer to $e, \mu$, and $\tau$ leptons and the $q$ 's to $d, u, s, c$, and $b$ quarks. Finally, the main advantage of exploiting $\gamma \gamma$ collisions in place of $e^{+} e^{-}$ones $[10,11]$ in producing single sneutrinos in association with a fermion pair in final states of the type (2) resides in the fact that the cross sections for the former are generally larger than those for the latter, as one can appreciate in Fig. 1. There, as an illustration, we have plotted the unpolarized production rates for both the $\gamma \gamma$ and $e^{+} e^{-}$induced modes, using the photon structure functions given in [2], at $\sqrt{s_{e^{+} e^{-}}}=500 \mathrm{GeV}$ and 1 TeV. Apart from the $\tilde{\nu} e^{+} e^{-}$final state, which in electronpositron annihilation receives very large additional contributions from small angle Bhabha-like scattering amplitudes (with respect to the other final states), the photon processes are dominant over the electron-positron ones. ${ }^{1}$

The $\gamma \gamma$ induced associate production process has been investigated recently in Ref. [13] by assuming unpolarized photon beams and without any detailed background estimates. We will improve on that study by exploiting polarized $\gamma \gamma$ scatterings, as it has been shown that a high degree of polarization can be transmitted from the electrons, positrons, and laser photons to the Compton backscattered photons, and by including a study of the irreducible SM background. ${ }^{2}$ In fact, it will be shown that polarization may help to improve the signal-to-background ratio $(S / B)$ in some instances. We

\footnotetext{
${ }^{1}$ We will defer the study of the $e^{+} e^{-}$processes to another paper [14]. We should, however, mention here that we have verified that, given the final luminosities collected at the CERN $e^{+} e^{-}$collider (see Ref. [15]), the signatures considered in Eqs. (2) and (3) but produced via $e^{+} e^{-}$annihilations between 206 and $210 \mathrm{GeV}$ could have not been seen at the CERN machine, for the choice of RPV couplings adopted in the following (see also [16]).

${ }^{2}$ We make use of HELAS [17] and MADGRAPH [18] to produce the helicity amplitudes, for both signal and backgrounds, and integrate these numerically by using VEGAS [19].
} 
TABLE II. Set of selected points in the MSSM parameter space with LSP and lighter chargino mass (and nature) given explicitly (we defer to the Appendix the listing of the total decay widths of the sneutrino in different RPV channels for these three choices of MSSM parameters).

\begin{tabular}{lcccccc}
\hline \hline Set & $\mu(\mathrm{GeV})$ & $M_{2}(\mathrm{GeV})$ & $\tan \beta$ & $M_{\tilde{\chi}_{1}^{0}}(\mathrm{GeV})$ & $M_{\tilde{\chi}_{1}^{ \pm}}(\mathrm{GeV})$ & Nature of $\tilde{\chi}_{1}^{ \pm}$ \\
\hline A & -400 & 150 & 5 & 76.4 & 150.3 & Gaugino dominated state \\
B & 200 & 350 & 40 & 150.4 & 185.6 & mixed state \\
C & 175 & 500 & 40 & 155.6 & 169.4 & Higgsino dominated state \\
\hline \hline
\end{tabular}

consider a general MSSM parameter space, with no assumption on the mechanism of SUSY breaking, hence defining all parameters at the EW scale.

Before proceeding to the analysis, it is is useful to note at this point that the $\epsilon_{i}$ terms in Eq. (1) can in principle be removed by a redefinition of the lepton doublets $\hat{L}_{i}$, which would in turn lead to their "absorption" into the $\lambda, \lambda^{\prime}$ couplings and in the parameters of the scalar potential of the SUSY model. However, the $\epsilon_{i}$ 's could then reappear at a different energy scale. Bilinear terms could also lead to a possible vacuum expectation value (VEV) for the sneutrino(s) and mixing of (a) charged leptons with charginos, (b) sleptons with charged Higgs bosons, (c) neutrinos with neutralinos, and (d) sneutrinos with neutral Higgs bosons. This last mixing could indeed affect the process discussed here. However, this phenomenon is suppressed by the small Yukawa couplings of our $l$ and $q$ fermions, so that we feel justified in neglecting it here (i.e., we are making the assumption that the $\epsilon_{i}$ terms are small). ${ }^{3}$

The paper is organized as follows. In Sec. III, we discuss the phenomenology of processes (2) and (3) in presence of polarized incoming photons. In Sec. IV we present our numerical results (including those for the backgrounds), followed by our conclusions in Sec. V.

\section{SINGLY PRODUCED SNEUTRINOS AT POLARIZED PHOTON COLLIDERS}

In the RPV MSSM, the sneutrino displays a coupling with pairs of leptons ( $\lambda$-type couplings) and quarks ( $\lambda^{\prime}$-type couplings). Single production of sneutrino in association with fermion pairs in Eq. (2) can occur through any of these two types of L-violating couplings. Depending upon the nature of the vertex involved, the above process may also lead to flavor changing final states.

The polarized photon flux and polarization have been worked out in [2] and are discussed in detail in Ref. [3]. For brevity, we do not reproduce here those formulas, rather we simply recall to the unfamiliar reader the basic features of polarized $\gamma \gamma$ scatterings.

(1) We assume that the laser backscattering parameter as-

\footnotetext{
${ }^{3}$ This would not be possible for processes involving top (anti)quarks, because of their large mass. However, in Eqs. (2) and (3), $t$ quarks contributions will have negligible impact because they are strongly suppressed by phase space effects. (Some phenomenological consequences of a sneutrino VEV and L-violating mixing have been discussed in literature [20].)
}

sumes its maximum value, $z \equiv z_{\max }=2(1+\sqrt{2}) \simeq 4.828$ [2]. In fact, with increasing $z$ the high energy photon spectrum becomes more monochromatic. However, for $z>z_{\max }$, the probability of $e^{+} e^{-}$pair creation increases, resulting in larger photon beam degradation.

(2) The reflected photon beam carries off only a fraction $x$ of the $e^{ \pm}$energy, with $x_{\max }=z /(1+z) \simeq 0.8$, while $x_{\min }=\left(M_{\tilde{\nu}}\right.$ $\left.+m_{f}+m_{f^{\prime}}\right) / \sqrt{s_{e^{+} e^{-}}}$(hereafter, $\left.f^{(')}=l, q\right)$.

(3) The polarization of the two initial laser $(\gamma)$ and electron/positron $(e)$ beams are defined by $P_{\gamma_{-}}, P_{\gamma_{+}}, P_{e^{-}}$, and $P_{e^{+}}$, respectively, where, for the first two quantities, $-(+)$ identifies the laser colliding against the electron (positron).

(4) Finally, one can cast the polarized production cross section in the following form:

$$
\begin{aligned}
\sigma_{e^{+} e^{-} \rightarrow \gamma \gamma \rightarrow \tilde{\nu} f \bar{f}^{\prime}}(s)= & \int d x_{-} d x_{+} F_{-}^{\gamma / e}\left(P_{e^{-}}, P_{\gamma_{-}}, x_{-} ; P_{-}\right) \\
& \times F_{+}^{\gamma / e}\left(P_{e^{+}}, P_{\gamma_{+}}, x_{+} ; P_{+}\right) \hat{\sigma}_{\gamma \gamma \rightarrow \tilde{\nu} f \bar{f}^{\prime}} \\
& \times\left(\hat{s}, P_{-}, P_{+}\right)
\end{aligned}
$$

where $x_{-(+)}$is the electron(positron) momentum fraction carried by the emerging photon, $x_{-} x_{+}=\hat{s}_{\gamma \gamma} / s_{e^{+} e^{-}}$, with

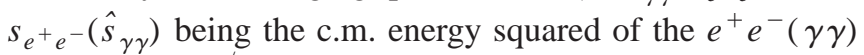
system, and $F_{ \pm}^{\gamma / e}\left(P_{e^{ \pm}}, P_{\gamma_{ \pm}}, x_{ \pm} ; P_{ \pm}\right)$the photon distribution functions, defined in terms of $P_{e^{ \pm}}, P_{\gamma_{+}}$, and $x_{ \pm}$and yielding $P_{-}\left(P_{+}\right)$, the degree of polarization of the photon that has backscattered against the electron (positron). ${ }^{4}$ Therefore in terms of helicity amplitudes one has (here, for brevity, $\hat{\sigma}$ $\left.\equiv \hat{\sigma}_{\gamma \gamma \rightarrow \tilde{\nu} f \bar{f}^{\prime}}\right)$

$$
\begin{aligned}
\hat{\sigma}\left(\hat{s}, P_{-}, P_{+}\right)= & \frac{1}{4}\left[\left(1+P_{-}\right)\left(1+P_{+}\right) \hat{\sigma}_{++}(\hat{s})\right. \\
& +\left(1+P_{-}\right)\left(1-P_{+}\right) \hat{\sigma}_{+-}(\hat{s})+\left(1-P_{-}\right) \\
& \times\left(1+P_{+}\right) \hat{\sigma}_{-+}(\hat{s}) \\
& \left.+\left(1-P_{-}\right)\left(1-P_{+}\right) \hat{\sigma}_{--}(\hat{s})\right] .
\end{aligned}
$$

\footnotetext{
${ }^{4}$ Conventionally, one has $P_{-(+)}=-1(+1)$ for purely left(right-) handed photons.
} 
TABLE III. Values of laser and electron (positron) beam polarizations adopted in our analysis. The $\sigma(+-)$ and $\sigma(++)$ denote the corresponding polarized production cross sections, with $\sigma(00)$ the unpolarized one.

\begin{tabular}{lcccc}
\hline \hline & $P_{\gamma_{+}}$ & $P_{\gamma_{-}}$ & $P_{e^{+}}$ & $P_{e^{-}}$ \\
\hline$\sigma(+-)$ & +1 & -1 & -0.8 & +0.9 \\
$\sigma(++)$ & +1 & +1 & -0.8 & -0.9 \\
$\sigma(00)$ & 0 & 0 & 0 & 0 \\
\hline \hline
\end{tabular}

As polarized $\gamma$-structure functions we have used those of Ref. [21].

The flavor of the final state fermions will depend upon the RPV couplings involved. It has been shown that most of the first two generation L-violating terms are highly constrained from different low and medium energy processes [22]. For our study, we made the assumption that just one L-violating coupling at a time is the dominant one, so that only bounds derived under the same hypothesis are relevant. This restriction may seem unnatural, however, it is a useful approach that allows one to derive a quantitative feeling for the phenomenological consequences of RPV interactions, while avoiding a proliferation of SUSY input parameters. In our analysis we will concentrate on the following L-violating couplings: $\lambda_{311}, \lambda_{323}, \lambda_{323}^{\prime}$, and $\lambda_{333}^{\prime}$. The reason for selecting this particular set out of the 36 possible couplings is that these are less constrained and at the same time can lead to a significant contribution to the production as well as the decay rates of sneutrinos in Eqs. (2) and (3). The upper limits on these couplings and the processes which give such bounds are shown in Table I. Notice that all these limits scale as $M_{\tilde{f}} / 100 \mathrm{GeV}$ with the common sfermion mass. That is, they become weaker as $M_{\tilde{f}}$ increases. However, some couplings are constrained by the requirement of perturbative unitarity. For example, the corresponding bound on $\lambda_{323}^{\prime}$ is 1.12 . Indeed, we could have taken any values of these couplings bounded between the mentioned upper and lower limits (as done by [12]). However, like in [13] and for the sake of simplicity, we will consider only one fixed value for each of the RPV couplings, the one obtained assuming a $100 \mathrm{GeV}$ sfermion mass (as in Table I). In a sense then, our approach can be viewed as conservative.

Once the sneutrino is produced, it will decay. Depending on its nature, the dominant decay modes are

$$
\begin{aligned}
& \tilde{\nu} \rightarrow f \bar{f}^{\prime} \quad(f=l, q) \quad \text { fermion pairs, } \\
& \widetilde{\nu} \rightarrow \widetilde{\chi}_{i}^{0} \nu \quad(i=1,2,3,4) \quad \text { neutralino + neutrino, } \\
& \tilde{\nu} \rightarrow \tilde{\chi}_{i}^{+} l^{-} \quad(i=1,2) \quad \text { chargino + lepton. }
\end{aligned}
$$

If the sneutrino is the LSP, then it will decay through the first (RPV) channel, otherwise via one of the other two (MSSM) modes. We show the sneutrino branching ratio (BR) in two fermion final states in the $\mu-M_{2}$ plane for a fixed value of
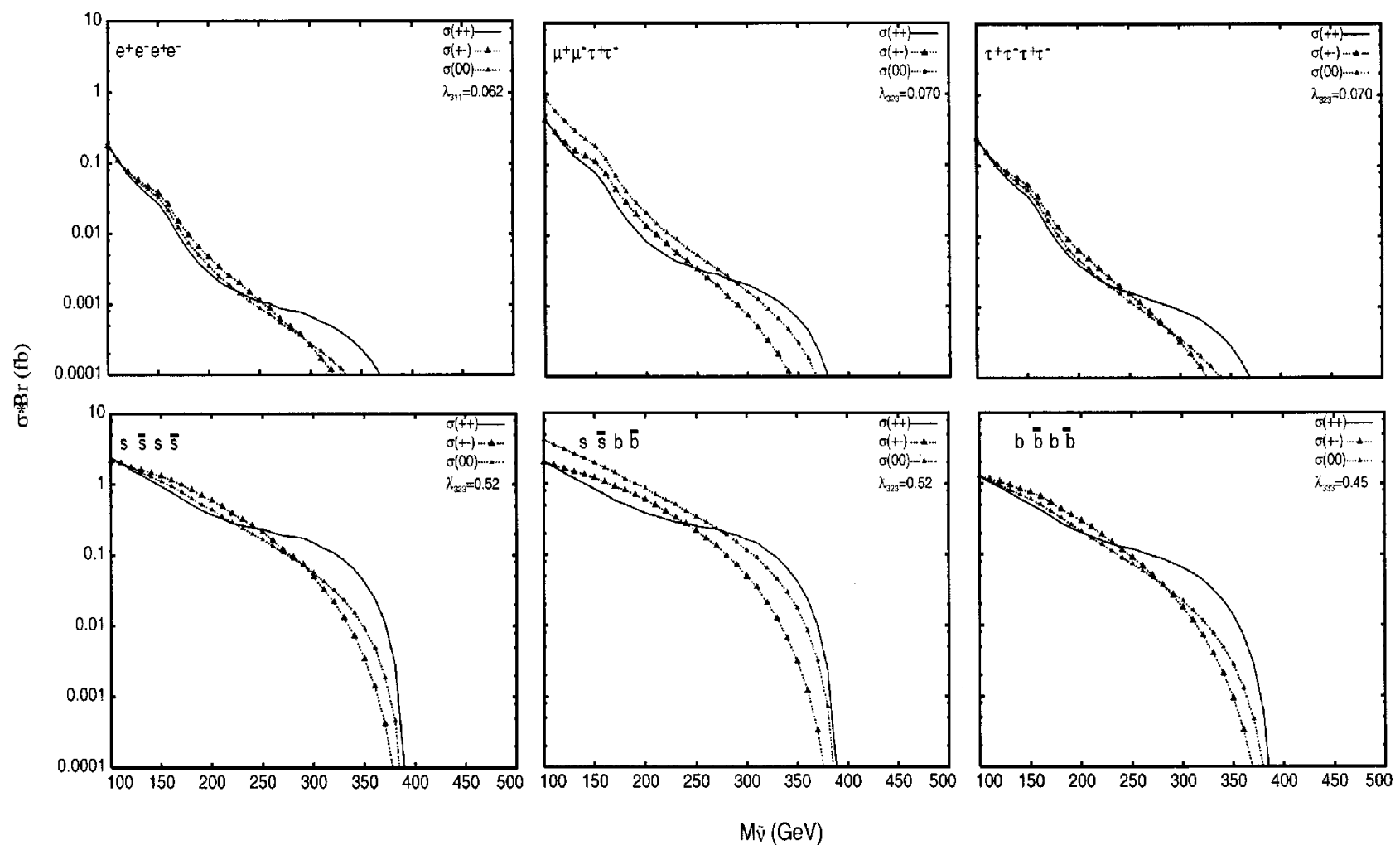

FIG. 3. Variation of $\sigma\left(\gamma \gamma \rightarrow \tilde{\nu}_{i} f_{j} \bar{f}_{k}\right) * \mathrm{BR}\left(\tilde{\nu}_{i} \rightarrow f_{j} \bar{f}_{k}\right)$ at $\sqrt{s_{e^{+} e^{-}}}=500 \mathrm{GeV}$ with the sneutrino mass, for fixed values of the relevant $\lambda_{i j k}$ and $\lambda_{i j k}^{\prime}$ couplings. The MSSM parameters are $\mu=-400 \mathrm{GeV}, M_{2}=150 \mathrm{GeV}$, and $\tan \beta=5$ (set A). Final state fermions are shown in each frame. For different flavors we include all charge conjugate states. See Table III for the definition of (un)polarized cross sections. 

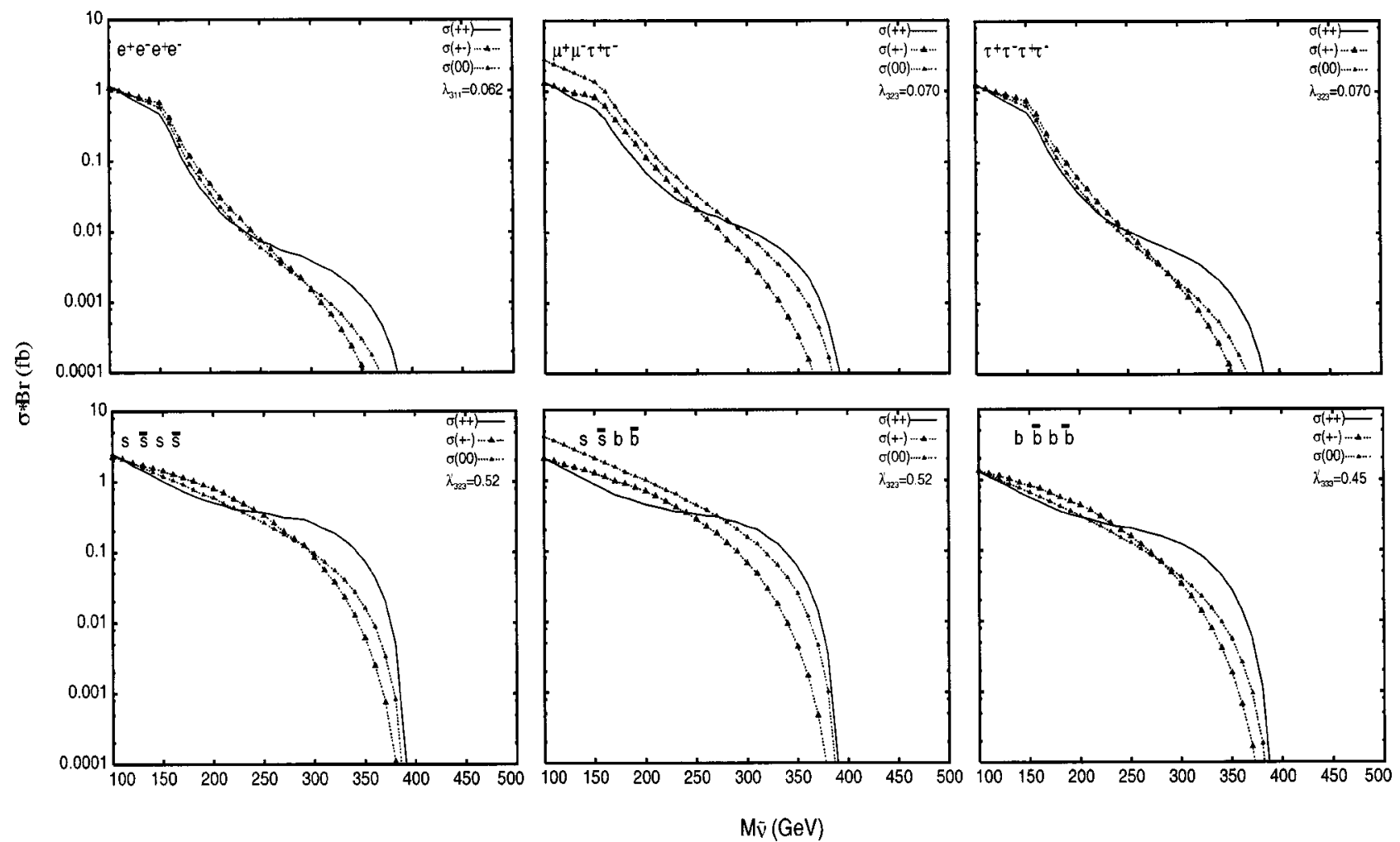

FIG. 4. Variation of $\sigma\left(\gamma \gamma \rightarrow \tilde{\nu}_{i} f_{j} \bar{f}_{k}\right) * \mathrm{BR}\left(\tilde{\nu}_{i} \rightarrow f_{j} \bar{f}_{k}\right)$ at $\sqrt{s_{e^{+} e^{-}}}=500 \mathrm{GeV}$ with the sneutrino mass, for fixed values of the relevant $\lambda_{i j k}$ and $\lambda_{i j k}^{\prime}$ couplings. The MSSM parameters are $\mu=200 \mathrm{GeV}, M_{2}=350 \mathrm{GeV}$, and $\tan \beta=40$ (set B). Final state fermions are shown in each frame. For different flavors we include all charge conjugate states. See Table III for the definition of (un)polarized cross sections.

$\tan \beta$, RPV coupling and sneutrino mass. In the course of the analysis we assume the grand unification (GUT) relationship between the $U(1)$ and $S U(2)$ gaugino mass parameters: i.e.,

$$
M_{1}=\frac{5}{3} \tan ^{2} \theta_{W} M_{2} .
$$

Hence the sneutrino BR into two fermions will depend upon $\mu, M_{2}, \tan \beta, M_{\tilde{\nu}}$, and the magnitude of the RPV coupling. To study the variation of the sneutrino RPV BR we have spanned $\mu$ from -500 to $500 \mathrm{GeV}$ and $M_{2}$ from 100 to 500 $\mathrm{GeV}$.

In Fig. 2(a) we show the contours of constant $\operatorname{BR}\left(\tilde{\nu}_{\tau}\right.$ $\rightarrow e^{+} e^{-}$) through the $\lambda_{311}$ coupling for $M_{\tilde{\nu}_{\tau}}=100 \mathrm{GeV}$ in the $\mu-M_{2}$ plane, with $\tan \beta=5$. The region labeled by "LEP disallowed" is ruled out from the kinematic limit on the lighter chargino mass extracted from LEP-2 data. It can be seen from this figure that the mentioned BR is $90 \%$ over a large part of the parameter space. In this case, the lighter chargino is heavier than the sneutrino mass, forbidding the $\tilde{\nu} \rightarrow \tilde{\chi}_{1}^{+} l^{-}$decay channel. The only MSSM channel allowed is $\tilde{\nu} \rightarrow \widetilde{\chi}_{1}^{0} \nu$, which dominates in the low $M_{2}$ region, where $M_{\tilde{\chi}_{1}^{0}}<M_{\tilde{\nu}}$. The above scenario changes once the sneutrino becomes heavier, as shown in Fig. 2(b), where the same BR as above is plotted but now with $M_{\tilde{\nu}}=200 \mathrm{GeV}$. In this case, both channels $\tilde{\nu} \rightarrow \tilde{\chi}_{1}^{+} l^{-}$and $\tilde{\nu} \rightarrow \tilde{\chi}_{1}^{0} \nu$ make a significant contribution to the total decay width of the sneutrino.
(The RPV BR increases with $M_{2}$ though, since the lighter chargino and neutralino become heavier.) In Fig. 2(c), this trend becomes very clear: for a $400 \mathrm{GeV}$ sneutrino most of the $\mu-M_{2}$ plane is covered by the MSSM decays, relegating large RPV BRs to small corners of the parameter space.

This situation changes considerably when the RPV coupling is $\lambda_{333}^{\prime}$. In this case, because of the larger magnitude of the latter, as compared to $\lambda_{311}$, the $\operatorname{BR}(\tilde{\nu} \rightarrow b \bar{b})$ for a 100 $\mathrm{GeV}$ sneutrino mass covers almost the entire $\mu-M_{2}$ plane analyzed in this paper. Even for heavier sneutrinos (e.g., 200 and $400 \mathrm{GeV}$ ), a larger area in the $\mu-M_{2}$ plane is dominated by the above BR, leaving a smaller region for the MSSM decays than in the previous case: see Figs. 2(d)-2(f). Finally, we have noticed that this general behavior of the BRs does not change for higher values of $\tan \beta$. Also, the impact of $\lambda_{323}$ and $\lambda_{323}^{\prime}$ RPV couplings onto the decay rates induces a pattern similar to the one discussed, so we do not reproduce the corresponding figures here.

\section{NUMERICAL ANALYSIS}

We perform our numerical analysis for three different points in the MSSM parameter space allowed by LEP-2 data. These are representative of three different natures of the lightest chargino and are defined in Table II.

Furthermore, we select the combinations of incident laser and electron beam polarizations shown in Table III.

The choice $P_{\gamma_{ \pm}} P_{e^{ \pm}}<0$ guarantees not only good mono- 

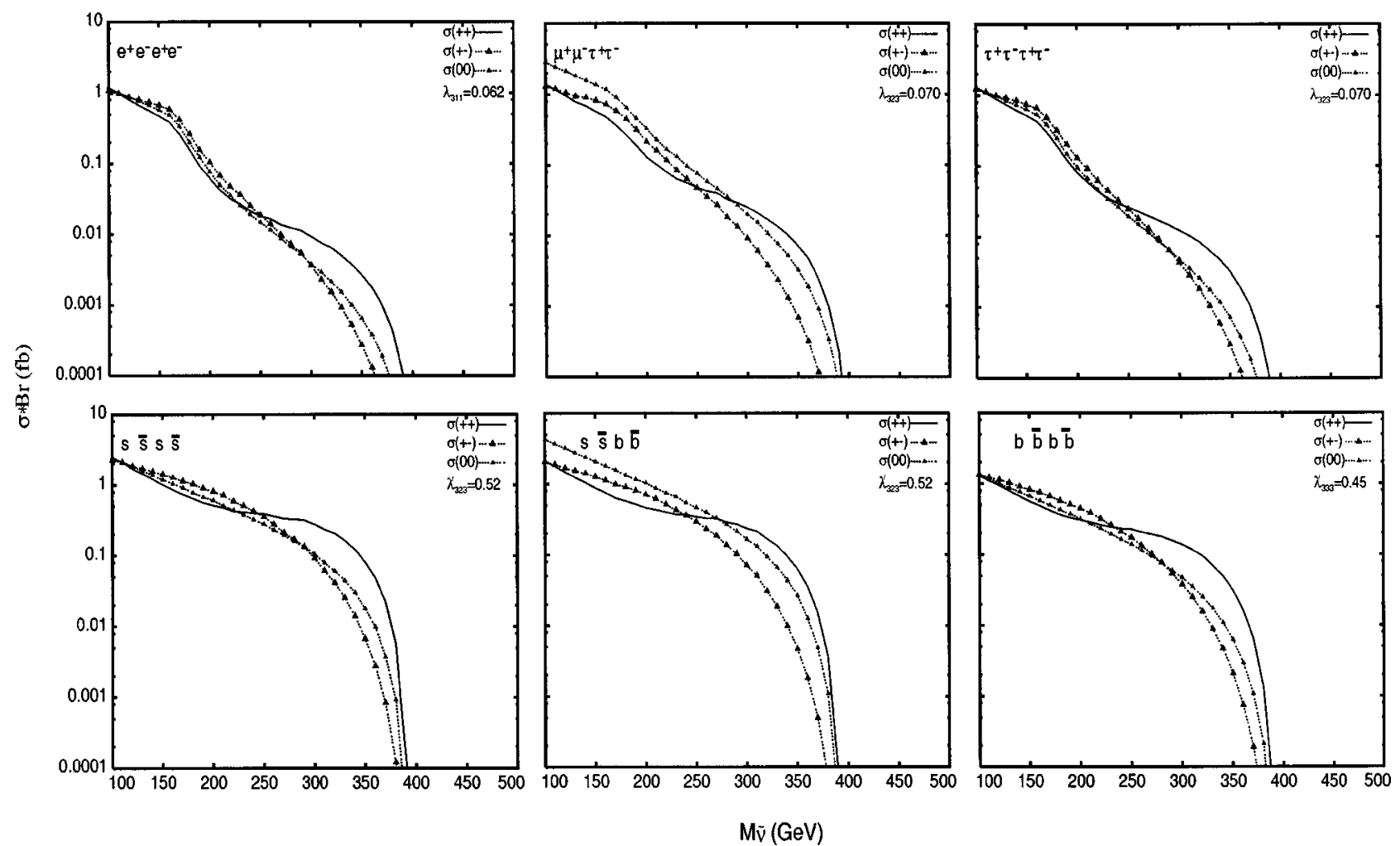

FIG. 5. Variation of $\sigma\left(\gamma \gamma \rightarrow \tilde{\nu}_{i} f_{j} \bar{f}_{k}\right) * \mathrm{BR}\left(\tilde{\nu}_{i} \rightarrow f_{j} \bar{f}_{k}\right)$ at $\sqrt{s_{e^{+} e^{-}}}=500 \mathrm{GeV}$ with the sneutrino mass, for fixed values of the relevant $\lambda_{i j k}$ and $\lambda_{i j k}^{\prime}$ couplings. The MSSM parameters are $\mu=175 \mathrm{GeV}, M_{2}=500 \mathrm{GeV}$, and $\tan \beta=40$ (set C). Final state fermions are shown in each frame. For different flavors we include all charge conjugate states. See Table III for the definition of (un)polarized cross sections.

chromaticity, but also a high degree of circular polarization of the produced photons as compared to the case $P_{\gamma_{+}} P_{e^{ \pm}}$ $>0$. There exists a symmetry amongst the four combinations of laser polarizations, as $(+-)$ and $(-+)$ give the same result, and so do $(++)$ and $(--)$ (see also [21]).

To mimic the finite coverage of the LC detectors, we impose the following cuts on the final state particles in Eq. (2): ${ }^{5}$

$$
5^{\circ}<\theta<175^{\circ} \quad \text { (angular cut on both leptons and jets) }
$$

$E_{l}>5 \mathrm{GeV} \quad$ (energy cut on leptons),

$$
E_{j}>10 \mathrm{GeV} \quad \text { (energy cut on jets). }
$$

As already mentioned, we assume that only one between the $\lambda$ and $\lambda^{\prime}$ couplings dominates at a time. Besides, we will treat the signatures arising from the four RPV couplings considered here, i.e., $\lambda_{311}, \lambda_{323}, \lambda_{323}^{\prime}$, and $\lambda_{333}^{\prime}$, separately in the four sections below. Where appropriate, all possible electromagnetic (EM) charge combinations (c.c.'s) will be included. Moreover, we assume that the EM charge of the leptons $(e, \mu$, and $\tau$ ) can always be determined, unlike the case of quarks. For the latter, we will assume a benchmark $100 \%$ efficiency in tagging $b$ flavors.

\footnotetext{
${ }^{5}$ We identify jets with the partons from which they originate.
}

\section{A. Signals from the $\boldsymbol{\lambda}_{311}$ coupling}

The presence of this coupling leads $\tilde{\nu}_{\tau}$ to decay into $e^{+} e^{-}$ pairs. Hence the signal corresponding to this L-violating coupling is $e^{+} e^{-} e^{+} e^{-}$. In Fig. 3(a) we show the variation of $\sigma\left(\gamma \gamma \rightarrow \tilde{\nu}_{\tau} e^{+} e^{-}\right) * \operatorname{BR}\left(\tilde{\nu}_{\tau} \rightarrow e^{+} e^{-}\right)$as a function of the $\tilde{\nu}_{\tau}$ mass for the MSSM set A, at $\sqrt{s_{e^{+} e^{-}}}=500 \mathrm{GeV}$. The effect of beam polarization can be seen very clearly from the figure. At very low sneutrino masses $(<150-200 \mathrm{GeV}), \sigma$ $(++), \sigma(+-)$ and the unpolarized cross section $\sigma(00)$ are basically the same. As the sneutrino mass rises, the above three cross sections display a hierarchy, though not dramatic, with $\sigma(+-)>\sigma(00)>\sigma(++)$, whereas for $M_{\tilde{\nu}_{\tau}}$ $\geqslant 0.5 \sqrt{s_{e^{+} e^{-}}}$, the $\sigma(++)$ component is the one which largely dominates. A similar situation can be seen for the other two sets of MSSM parameters, namely sets B and C, in Figs. 4(a) and 5(a) respectively. For $\sqrt{s_{e^{+} e^{-}}}=1 \mathrm{TeV}$, corresponding plots are given in Figs. 6(a), 7(a), and 8(a), for the MSSM parameter sets $\mathrm{A}, \mathrm{B}$, and $\mathrm{C}$, respectively. At higher energies, the pattern is very similar, with the only exceptions that in this case $\sigma(00)$ is slightly larger than the other two at small sneutrino masses and the mentioned hierarchy onsets for somewhat larger values of the latter, in comparison to the lower energy collider option.

\section{B. Signals from the $\boldsymbol{\lambda}_{323}$ coupling}

The presence of this coupling gives rise to the following two types of signals: flavor conserving $\tau^{+} \tau^{-} \tau^{+} \tau^{-}$and fla- 


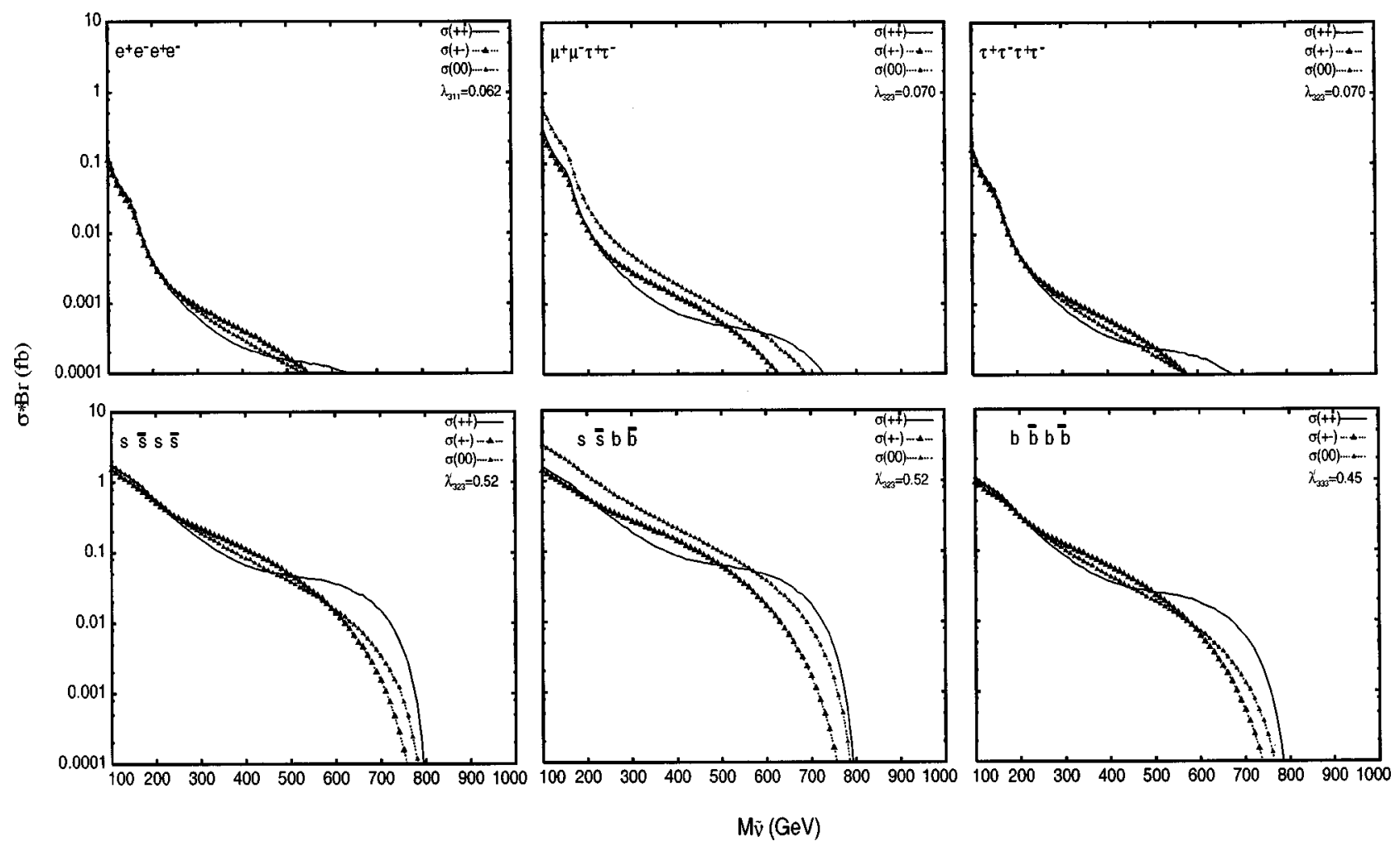

FIG. 6. Variation of $\sigma\left(\gamma \gamma \rightarrow \tilde{\nu}_{i} f_{j} \bar{f}_{k}\right) * \mathrm{BR}\left(\tilde{\nu}_{i} \rightarrow f_{j} \bar{f}_{k}\right)$ at $\sqrt{s_{e^{+} e^{-}}}=1 \mathrm{TeV}$ with the sneutrino mass, for fixed values of the relevant $\lambda_{i j k}$ and $\lambda_{i j k}^{\prime}$ couplings. The MSSM parameters are $\mu=-400 \mathrm{GeV}, M_{2}=150 \mathrm{GeV}$, and $\tan \beta=5$ (set A). Final state fermions are shown in each frame. For different flavors we include all charge conjugate states. See Table III for the definition of (un)polarized cross sections.

vor changing $\mu^{+} \mu^{-} \tau^{+} \tau^{-}$(and c.c.'s). The variation of $\sigma\left(\gamma \gamma \rightarrow \tilde{\nu}_{\tau} \mu^{+} \tau^{-}\right) * \operatorname{BR}\left(\tilde{\nu}_{\tau} \rightarrow \mu^{-} \tau^{+}\right)$as a function of the sneutrino mass is shown in Figs. 3(b), 4(b), and 5(b) for $\sqrt{s_{e^{+} e^{-}}}=500 \mathrm{GeV}$, and Figs. 6(b), 7(b), and 8(b) for $\sqrt{s_{e^{+} e^{-}}}=1 \mathrm{TeV}$, corresponding to the MSSM parameter sets $\mathrm{A}, \mathrm{B}$, and $\mathrm{C}$, respectively. In this case the final state will have three different combinations of charged particles with identical rates: $\mu^{+} \mu^{-} \tau^{+} \tau^{-}, \mu^{+} \mu^{+} \tau^{-} \tau^{-}$, and $\mu^{-} \mu^{-} \tau^{+} \tau^{+}$. Hence the individual channels will be $1 / 3$ of the total cross section shown in the figures. The plots for the flavor conserving final states are displayed in Figs. 3(c), 4(c) and 5(c), for $\sqrt{s_{e^{+} e^{-}}}=500 \mathrm{GeV}$, and Figs. 6(c), 7(c), and 8(c) for $\sqrt{s_{e^{+} e^{-}}}=1 \mathrm{TeV}$.

In this case too we see that the dominant cross section comes from $\sigma(++)$ once the $M_{\tilde{\nu}_{\tau}} \geqslant 0.5 \sqrt{s_{e^{+} e^{-}}}$. However, at lower sneutrino masses, the pattern is different from the previous case. The ordering $\sigma(+-)>\sigma(00)>\sigma(++)$ in the intermediate mass regime and the convergence of the rates for all polarization states at small $M_{\tilde{\nu}_{\tau}}$ values hold only for $\tau^{+} \tau^{-} \tau^{+} \tau^{-}$, not for $\mu^{+} \mu^{-} \tau^{+} \tau^{-}$(plus c.c.'s), for which the unpolarized cross sections are always largest. In this case, again, the increase in c.m. energy delays the onset of the highlighted cross section hierarchy, for $\tau^{+} \tau^{-} \tau^{+} \tau^{-}$final states.

\section{Signals from the $\boldsymbol{\lambda}_{323}^{\prime}$ coupling}

The presence of this coupling gives rise to the following three types of signals: the flavor conserving $s \bar{s} s \bar{s}$ and $b \bar{b} b \bar{b}$ plus the flavor changing $s \bar{s} b \bar{b}$ (and c.c.'s). The variation of $\sigma\left(\gamma \gamma \rightarrow \tilde{\nu}_{\tau} b \bar{s}\right) * \operatorname{BR}\left(\tilde{\nu}_{\tau} \rightarrow \bar{b} s\right)$ as a function of the sneutrino mass is shown in Figs. 3(e), 4(e), and 5(e) for $\sqrt{s_{e^{+} e^{-}}}$ $=500 \mathrm{GeV}$ and Figs. 6(e), 7(e), and 8(e) for $\sqrt{s_{e^{+} e^{-}}}$ $=1 \mathrm{TeV}$, again, in correspondence of the MSSM parameter sets $\mathrm{A}, \mathrm{B}$, and $\mathrm{C}$, respectively. Notice that in this case too there are three equiprobable signatures: $s s \bar{b} \bar{b}, s \bar{s} b \bar{b}$, and $\bar{s} \bar{s} b b$. Corresponding plots for the flavor conserving modes are displayed in Figs. 3(d), 4(d), and 5(d), for $\sqrt{s_{e^{+} e^{-}}}$ $=500 \mathrm{GeV}$ and Figs. 6(d), 7(d), and 8(d) for $\sqrt{s_{e^{+} e^{-}}}$ $=1 \mathrm{TeV}$ (in correspondence of sets $\mathrm{A}, \mathrm{B}$, and $\mathrm{C}$ ).

The dependence upon the beam polarization configuration is basically the same as the one described in the previous section, once one establishes a correspondence between the identical- and different-flavor final states in the two cases. The energy dependence does not differ much either from that in the two previous cases.

\section{Signals from the $\lambda_{333}^{\prime}$ coupling}

The presence of this coupling will also give rise to the signal $b \bar{b} b \bar{b}$. The numerical results for the corresponding production cross sections are shown in Figs. 3(f), 4(f), and 5(f) for $\sqrt{s_{e^{+} e^{-}}}=500 \mathrm{GeV}$ and Figs. 6(f), 7(f), and 8(f) for $\sqrt{s_{e^{+} e^{-}}}=1 \mathrm{TeV}$, corresponding to the MSSM parameter sets $\mathrm{A}, \mathrm{B}$, and $\mathrm{C}$, respectively.

As for the beam polarization dependence, here, one can see the usual dominance of $\sigma(++)$ whenever $M_{\tilde{\nu}_{\tau}}$ 


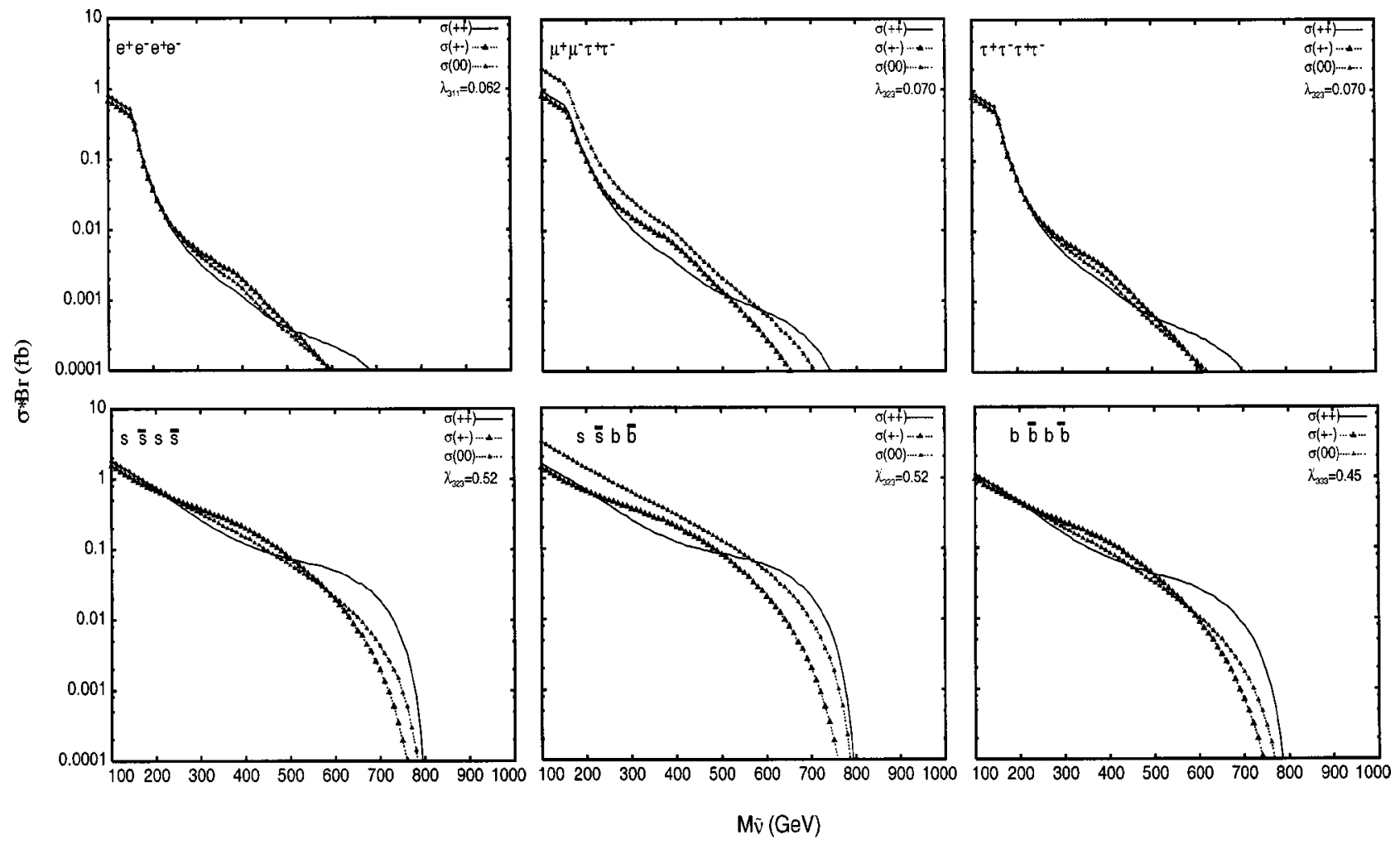

FIG. 7. Variation of $\sigma\left(\gamma \gamma \rightarrow \tilde{\nu}_{i} f_{j} \bar{f}_{k}\right) * \mathrm{BR}\left(\tilde{\nu}_{i} \rightarrow f_{j} \bar{f}_{k}\right)$ at $\sqrt{s_{e^{+} e^{-}}}=1 \mathrm{TeV}$ with the sneutrino mass, for fixed values of the relevant $\lambda_{i j k}$ and $\lambda_{i j k}^{\prime}$ couplings. The MSSM parameters are $\mu=200 \mathrm{GeV}, M_{2}=350 \mathrm{GeV}$, and $\tan \beta=40$ (set B). Final state fermions are shown in each frame. For different flavors we include all charge conjugate states. See Table III for the definition of (un)polarized cross sections.

$\geqslant 0.5 \sqrt{s_{e^{+} e^{-}}}$, with the $\sigma(+-)$ component dominating in the intermediate regime. For lower masses, the energy dependence is such that at $500 \mathrm{GeV} \sigma(+-)$ is above $\sigma(00)$, whereas at $1 \mathrm{TeV}$ things go the other way around.

\section{E. Signals from $\tilde{\nu} \rightarrow \tilde{\chi}_{1}^{+} l^{-}$}

Here, we would like to comment about the signal cross section $\sigma(\gamma \gamma \rightarrow \tilde{\nu} f \bar{f}) * \mathrm{BR}\left(\tilde{\nu} \rightarrow \tilde{\chi}_{1}^{+} l^{-}\right)$for two different RPV interactions, namely $\lambda_{311}$ and $\lambda_{323}^{\prime}$. Figures 9(a)-9(c) correspond to $\sigma\left(\gamma \gamma \rightarrow \tilde{\nu}_{\tau} e^{+} e^{-}\right) * \operatorname{BR}\left(\tilde{\nu}_{\tau} \rightarrow \tilde{\chi}_{1}^{+} \tau^{-}\right)$for $\lambda_{311}$ $=0.062$ whereas the variation of $\sigma(\gamma \gamma \rightarrow \tilde{\nu} s \bar{b}) * \operatorname{BR}\left(\tilde{\nu}_{\tau}\right.$ $\rightarrow \tilde{\chi}_{1}^{+} \tau^{-}$) with the sneutrino mass (for $\lambda_{323}^{\prime}=0.52$ ) is shown in Figs. 9(d)-9(f). Notice that Figs. 9(a,d), 9(b,e), and 9(c,f) correspond to the three usual sets of MSSM parameters A, B, and $\mathrm{C}$, respectively. These cross sections have been calculated for the case of a LC of $500 \mathrm{GeV}$. The pattern of the production and decay rates is here quite different from the one displayed for the case of RPV decays of the sneutrino. In fact, the overall behavior in this channel depends on other factors. First, on the relative mass difference between $\tilde{\nu}$ and $\tilde{\chi}_{1}^{+}$, as well as upon the composition of $\chi_{1}^{+}$(if it is Higgsino dominated, then the $\tilde{\nu}-\tilde{\chi}_{1}^{+}-l^{-}$coupling will be Yukawa suppressed). Second, and most importantly, the magnitude of the RPV coupling involved: as it is clear from comparing Figs. 9(a)-9(c) to Fig. 9(d)-9(f), the stronger the RPV coupling the smaller the $\tilde{\nu} \rightarrow \tilde{\chi}_{1}^{+} l^{-}$decay mode. In other words, this signal is somehow complementary to the RPV ones discussed so far and requires a different discussion of the decay dynamics, given the additional dependence on the chargino mass. Hence although this signature may well induce visible events in the end, we do not pursue further its study here.

\section{F. The SM irreducible background}

If $M_{\tilde{\nu}}$ is very near the EW scale, say, $80-90 \mathrm{GeV}$, it is clear that the dominant SM irreducible background to RPV signals of the type discussed in the previous sections arises from associated production of a $Z$ boson and a fermionic pair, with the gauge boson decaying into two further fermions:

$$
\gamma \gamma \rightarrow Z l^{ \pm} l^{\mp} \quad \text { or } \quad Z q \bar{q}
$$

with

$$
Z \rightarrow l^{\prime}{ }^{ \pm} l^{\prime \mp} \quad \text { or } \quad Z \rightarrow q^{\prime} \bar{q}^{\prime} .
$$

Only in the case of four-quark final states has one to deal with $W^{ \pm}$mediated production:

$$
\gamma \gamma \rightarrow W^{ \pm} q \bar{q}^{\prime}
$$

with

$$
W^{ \pm} \rightarrow q^{\prime \prime} \bar{q}^{\prime \prime \prime}
$$



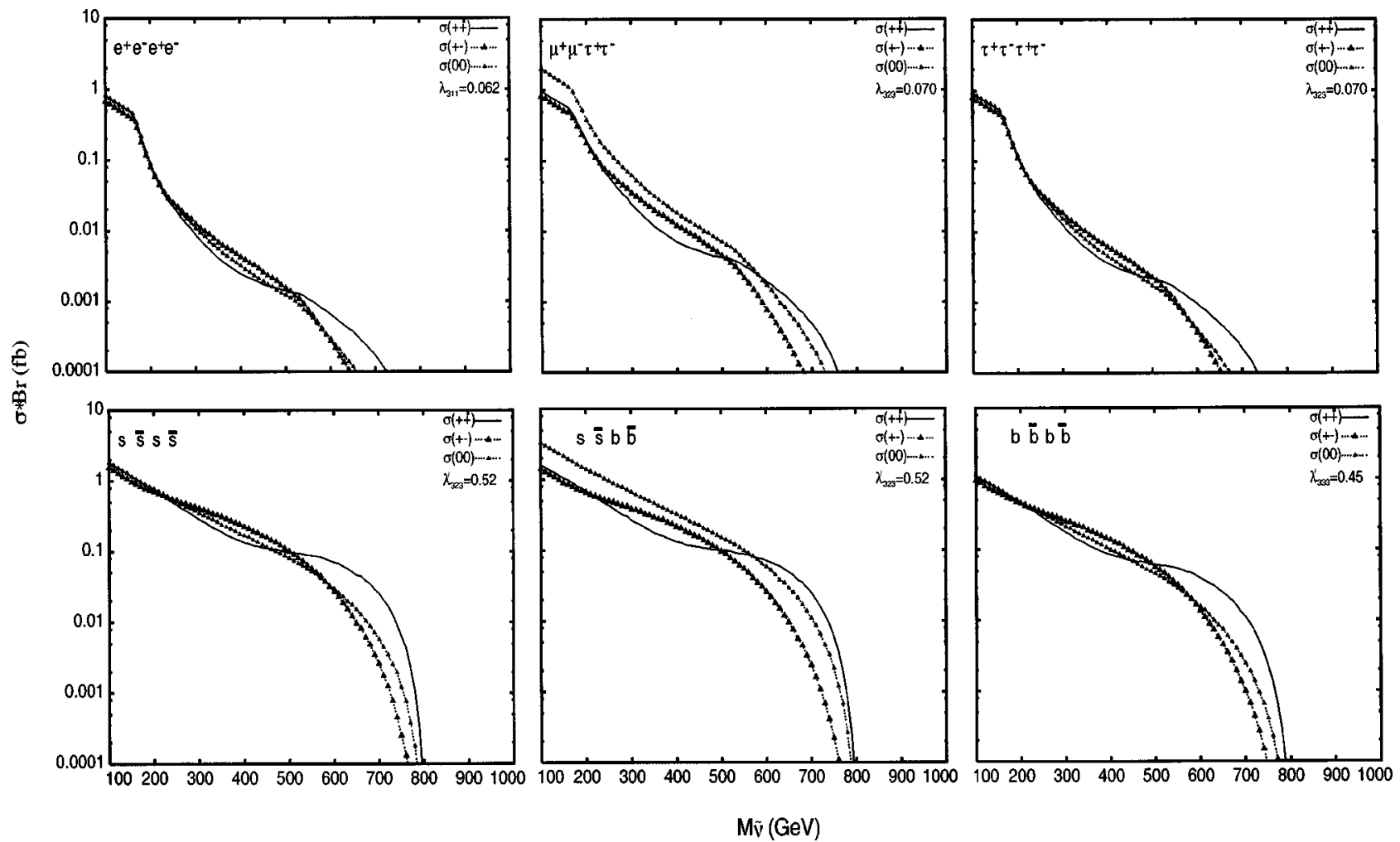

FIG. 8. Variation of $\sigma\left(\gamma \gamma \rightarrow \tilde{\nu}_{i} f_{j} \bar{f}_{k}\right) * \mathrm{BR}\left(\tilde{\nu}_{i} \rightarrow f_{j} \bar{f}_{k}\right)$ at $\sqrt{s_{e^{+} e^{-}}}=1 \mathrm{TeV}$ with the sneutrino mass, for fixed values of the relevant $\lambda_{i j k}$ and $\lambda_{i j k}^{\prime}$ couplings. The MSSM parameters are $\mu=175 \mathrm{GeV}, M_{2}=500 \mathrm{GeV}$ and $\tan \beta=40$ (set C). Final state fermions are shown in each frame. For different flavours we include all charge conjugate states. See Table III for the definition of (un)polarized cross sections.

However, notice that, with the exception of the $\bar{s} \bar{s} \bar{s}$ signature, only Cabibbo-Kobayashi-Maskawa (CKM) suppressed channels can contribute in Eqs. (15) and (16) if one assumes a fully efficient heavy quark tagging (via a displaced vertex) to be available at future LCs (i.e., $\boldsymbol{\epsilon}_{c, b}$ $=100 \%)$. This is precisely what occurs in the case of $s \bar{s} b \bar{b}$ final states whereas $W^{ \pm}$mediated SM backgrounds cannot contribute to $b \bar{b} b \bar{b}$ final states under the above assumption (we will briefly discuss the more realistic scenario arising from a finite efficiency for the latter in the last section).

When the sneutrino mass starts departing from $M_{Z}$ (or $M_{W}$ ), then a variety of SM subprocesses could produce sizable irreducible backgrounds, although at very heavy $M_{\tilde{\nu}}$ values only the tails of the SM distributions can actually play a role. All these channels can be conveniently grouped into general four-fermion final states, of the type $e^{+} e^{-} e^{+} e^{-}$, $\mu^{+} \mu^{-} \tau^{+} \tau^{-}, \tau^{+} \tau^{-} \tau^{+} \tau^{-}, s \bar{s} s \bar{s}, s \bar{s} b \bar{b}$, and $b \bar{b} b \bar{b}$, which we have generated by means of all Feynman graphs appearing at leading order, with the only exclusion of Higgs mediated graphs which are irrelevant for the first four channels because of the smallness of the Yukawa couplings involved (see also footnote 6), and since they can easily be excluded in the last two cases via a suitable invariant mass cut, i.e., $M_{b \bar{b}} \neq M_{\text {Higgs }}$, for any known neutral Higgs mass state of the model thanks to the narrowness of the Higgs boson resonances below the $W^{ \pm} W^{\mp *}$ threshold.

The first three signatures only receive EW contributions [i.e., they are of $\mathcal{O}\left(\alpha^{4}\right)$ ], whereas the last three also have a QCD induced component of $\mathcal{O}\left(\alpha^{2} \alpha_{s}^{2}\right)$. Under the assump- tion of perfect $b$ (and $c$ ) quark tagging, we only need to sum over light quark flavors in the case of $s \bar{s} s \bar{s}$ and $s \bar{s} b \bar{b}$ final states, not of $b \bar{b} b \bar{b}$. However, in order to save computing time, we have presently refrained from doing so, as the EW and QCD contributions involving only $s$ (anti)quarks, as opposed to the sum over $u, d$, and $s$, already swamp the signal in the accessible $M_{\tilde{\nu}}$ regions. (All forthcoming $s \bar{s} s \bar{s}$ and $s \bar{s} b \bar{b}$ background rates will then be reported for $s$ flavors only. $)^{6}$ Of course, four-fermion final states computed this way also include the contributions of processes of the type (13) and (14) and (15) and (16).

The SM background cross sections, after the cuts listed in Eq. (10), are given in Table IV. A common feature to all rates is that they are basically independent of the polarization state of the initial particles. ${ }^{7}$ By comparing the background rates in Table IV to those for the signals in Figs. 3-8, it is clear

\footnotetext{
${ }^{6}$ Also, we have used the two-loop expression for $\alpha_{s}$ as a function of the energy scale $Q \equiv \sqrt{s_{\gamma \gamma}}$ and of $\Lambda_{\mathrm{QCD}}^{n_{f}=4}=0.230 \mathrm{GeV}$.

${ }^{7}$ The huge rates for the $e^{+} e^{-} e^{+} e^{-}$final state should not be surprising: on the one hand, because of the $t, u$ channel soft and collinear singularities of the total cross section (which are only regulated by the small electron mass); on the other hand, since we have implemented rather loose constraints in order to avoid these, i.e., other than the cuts in individual energy and polar angles of Eq. (10) we only required $M_{e^{+} e^{-}}>1 \mathrm{GeV}$ on all electron-positron pairs (this combination is sufficient to obtain a numerically stable answer).
} 

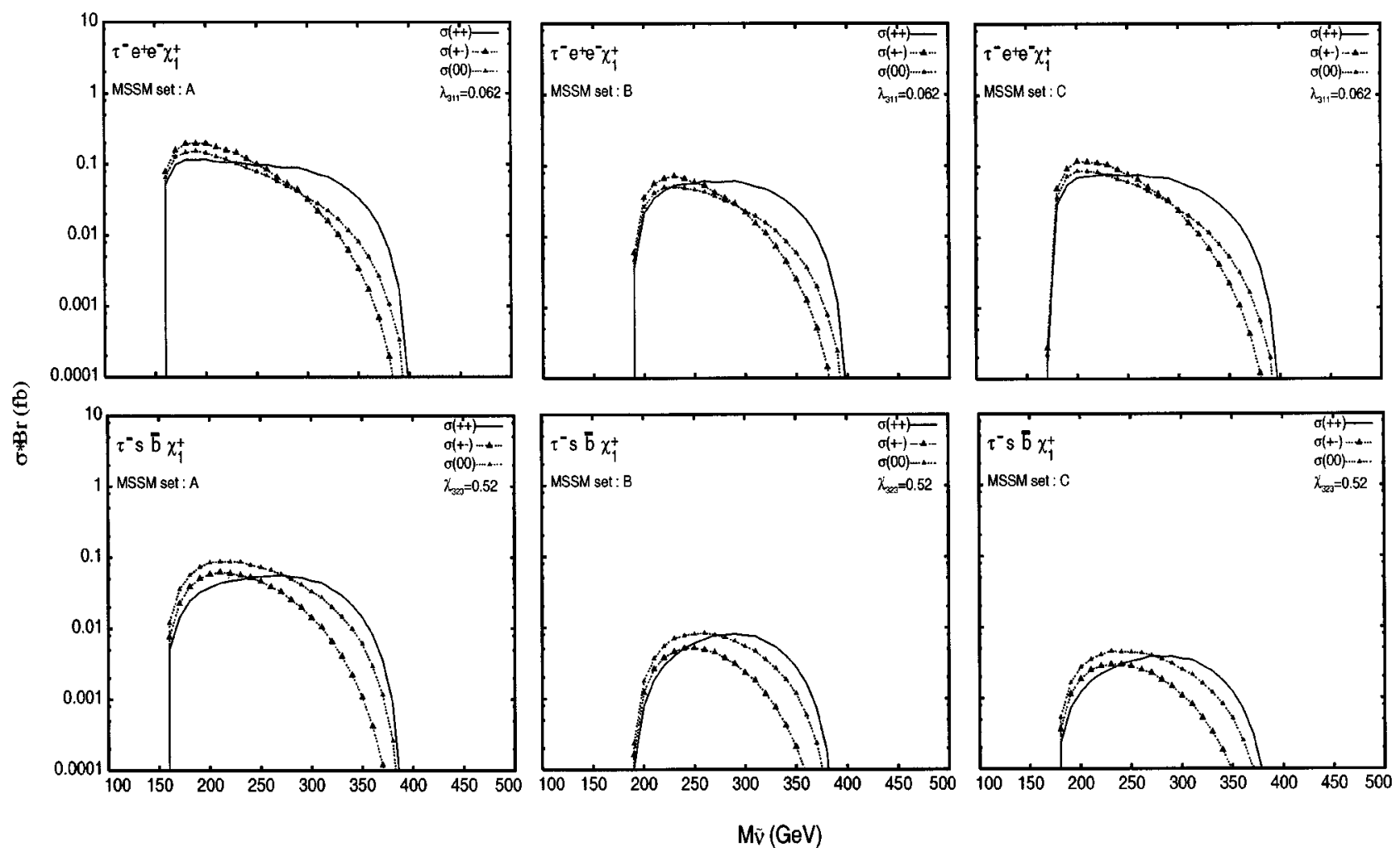

FIG. 9. Variation of $\sigma\left(\gamma \gamma \rightarrow \tilde{\nu}_{i} f_{j} \bar{f}_{k}\right) * \mathrm{BR}\left(\tilde{\nu}_{i} \rightarrow l^{-} \tilde{\chi}_{1}^{+}\right)$at $\sqrt{s_{e^{+} e^{-}}}=500 \mathrm{GeV}$ with the sneutrino mass, for fixed values of the relevant $\lambda_{i j k}$ and $\lambda_{i j k}^{\prime}$ couplings. MSSM parameter sets are shown in each frame. Beam polarization conventions are the same as in the previous figures.

that the former are overwhelming the latter in the inclusive cross sections. However, several selection cuts can be applied in order to improve the signal-to-background ratio $(S / B)$. For example, the $Z$ mediated noise in four-lepton final states can be reduced by requiring that no $l^{+} l^{-}$pairs of opposite charge (with $l=e, \mu$ ) reproduce the $Z$ mass within a few $\mathrm{GeV}$ (say, 3 or $4 \mathrm{GeV}$, given the good mass resolution expected at LCs for electrons and muons). Similarly, one can proceed for four-quark final states by rejecting events with one (or more) jet-jet invariant masses in the vicinity of the $Z$ and $W^{ \pm}$peaks. The $4 \tau$ signature is more difficult to deal with in this respect because of the missing momentum carried away by the neutrinos. Finally, QCD induced four-jet backgrounds tend to produce at least one jet-jet pair with small invariant mass.

In the very end, however, one should keep in mind that we are dealing with sneutrino masses that are bound by current experimental constraints to be above the EW scale. Hence, in general, by restricting oneself to the most interesting mass range, sufficiently far from the $Z$ and $W^{ \pm}$masses, say, 100-150 GeV or above, the chances of extracting the RPV signals in some of the channels discussed become evident, if one refers to Fig. 10 and to the rates in Figs. 3-8.

By finally recalling that sneutrinos yield mass resonances that are rather narrow (see the typical widths in the Appendix), one can further enhance the $S / B$ by restricting the candidate sample around the resonances. To this end, we present Tables V and VI, where, alongside the signal yield, the surviving background rates are given after we have required that only one (di-lepton or di-jet) invariant mass reconstructs the resonant sneutrino mass within $10 \mathrm{GeV}$. Notice that we have accounted for all combinatorics in both leptonic and hadronic final states, assuming EM charge recognition in the former but not in the latter. Results are given for the unpolarized case for the sake of illustration. (In the case of polarized initial states, the pattern is very similar.)

At the end of this selection, one should expect the final states $\mu^{+} \mu^{-} \tau^{+} \tau^{-}, \tau^{+} \tau^{-} \tau^{+} \tau^{-}$, and $b \bar{b} b \bar{b}$ to achieve a significance $\sigma \equiv S / \sqrt{B}$ larger than 5 after $1 \mathrm{ab}^{-1}$ of luminosity in the region $M_{\tilde{\nu}} \sim 100-150 \mathrm{GeV}$, at both $\sqrt{s_{e^{+} e^{-}}}$ $=500 \mathrm{GeV}$ and $1 \mathrm{TeV}$, for all MSSM parameter sets considered in the case of the hadronic signature and limitedly to set $\mathrm{B}$ and $\mathrm{C}$ for the leptonic ones. The overall signal rates at that luminosity are about 1000 events per channel. At the higher collider energy option, an evidence (i.e., $\sigma \geq 3$ ) of the $4 b$ signal may appear also in the $M_{\tilde{\nu}} \sim 200-250 \mathrm{GeV}$ interval, at least for the MSSM sets B and C, with overall signal rates of order 500 events. All other signatures appear instead hopeless. Tables VII and VIII summarize our findings in this respect. The typical signal would then be an excess of $\mu^{+} \mu^{-} \tau^{+} \tau^{-}, \tau^{+} \tau^{-} \tau^{+} \tau^{-}$plus $b \bar{b} b \bar{b}$ events above the SM expectations for the corresponding four-fermion processes, with the bulk of the events centered in a rather narrow lepton-lepton or jet-jet mass region corresponding to the sneutrino mass.

\section{CONCLUSIONS}

Although a full Monte Carlo simulation, including all signals and backgrounds that we have discussed and in presence 


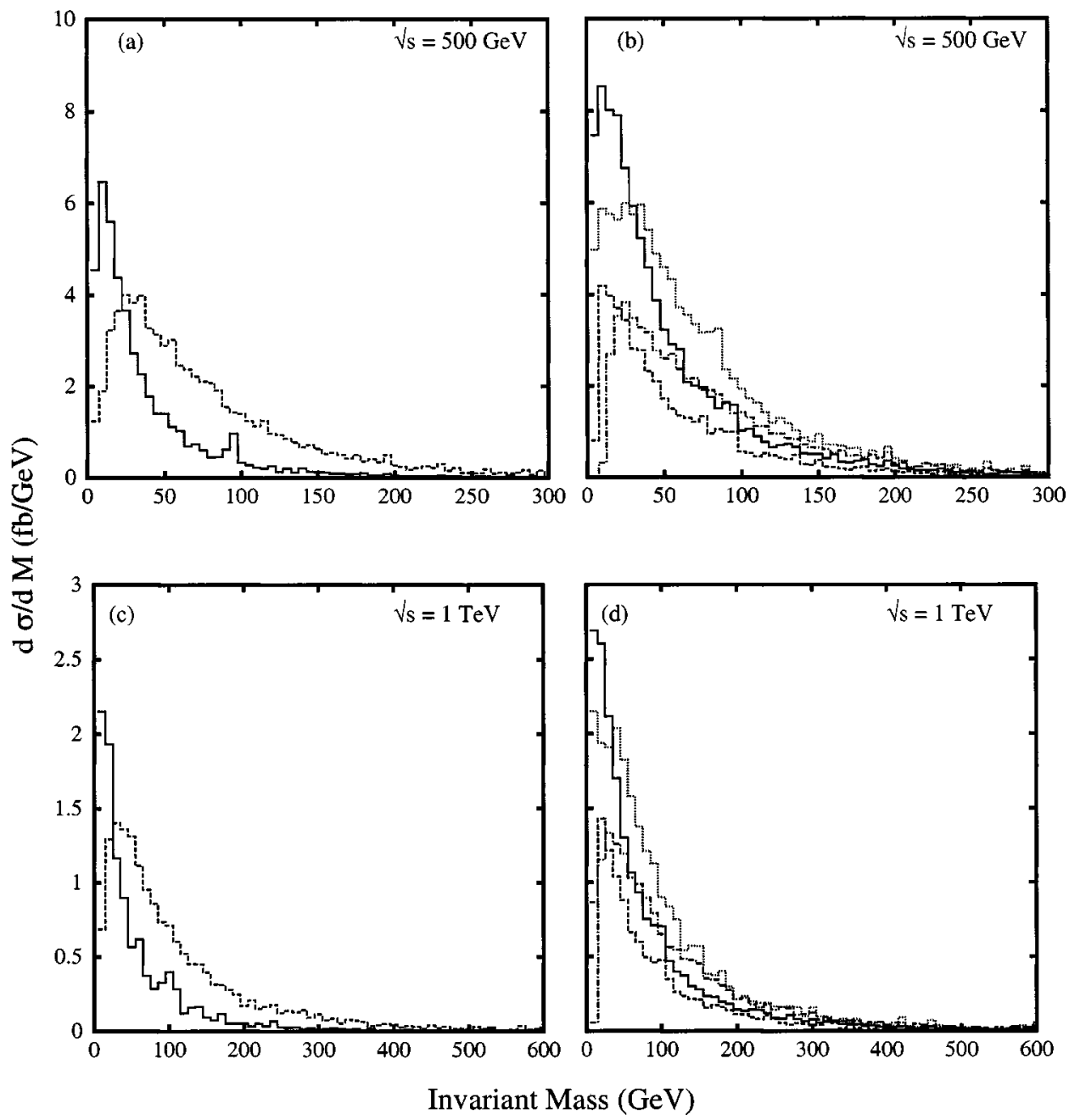

FIG. 10. Invariant mass distributions in lepton-lepton and jet-jet pairs reproducing the sneutrino mass in the background processes discussed in the text, for the case of unpolarized beams, $\sqrt{s_{e^{+} e^{-}}}$ $=500 \mathrm{GeV}$ and $1 \mathrm{TeV}$, after the cuts in Eq. (10) (the additional constraint $M_{e^{+} e^{-}}>1 \mathrm{GeV}$ has been implemented for the $e^{+} e^{-} e^{+} e^{-}$signature, for all possible pairings with opposite EM charge). Normalization is to the corresponding total cross sections given in Table IV. All possible combinatorial combinations have been plotted, each with the same probability, given by the event weight divided by the number of possible pairings in each case. Bins are $10 \mathrm{GeV}$ wide. Scaling factors have to be applied in order to obtain the original cross sections, as follows: (a,c) $\mu^{+} \mu^{-} \tau^{+} \tau^{-}$(solid) to be scaled by $10^{-2}, s \bar{s} b \bar{b}$ (dashed) by $10^{-2}$; (b,d) $e^{+} e^{-} e^{+} e^{-}$(solid) by $10^{4}$, $\tau^{+} \tau^{-} \tau^{+} \tau^{-}$(dashed) by $10^{-3}$, $s \bar{s} s \bar{s}$ (dotted) no scaling; and $b \bar{b} b \bar{b}$ (dot-dashed) by $10^{-3}$.

of both hadronization and detector effects, should eventually be performed in order to put on firmer ground the results presented here, it is clear that the latter seem rather promising at present.

In practice, if RPV couplings of the type $\lambda_{311}, \lambda_{323}, \lambda_{323}^{\prime}$, or $\lambda_{333}^{\prime}$ are close to their current exclusion bounds, over sizable regions of the MSSM parameter space (particularly, for positive $\mu$ values), several four-fermion signatures induced by a sneutrino, with a mass $M_{\tilde{\nu}} \lesssim 150 \mathrm{GeV}$ if $\sqrt{s_{e^{+} e^{-}}}$ $=500 \mathrm{GeV}$ and $\lesssim 250 \mathrm{GeV}$ if $\sqrt{s_{e^{+} e^{-}}}=1 \mathrm{TeV}$, produced in association with a fermion pair and decaying itself into a second pair can be accessed with the photons produced via back-scattering against the primary electrons and positrons. The typical annual rate should be of several hundreds to a

TABLE IV. Cross sections in femtobarns for the full four-fermion SM processes discussed in the text, for the three beam polarization configurations in Table III, after the cuts in Eq. (10) (and $M_{e^{+} e^{-}}>1 \mathrm{GeV}$ for the $e^{+} e^{-} e^{+} e^{-}$final state). Notice that no summation over $u, d$, and $s$ (light) flavors has been performed in the case of signatures involving $s$ quarks.

\begin{tabular}{|c|c|c|c|c|c|c|}
\hline & $e^{+} e^{-} e^{+} e^{-}$ & $\mu^{+} \mu^{-} \tau^{+} \tau^{-*}$ & $\tau^{+} \tau^{-} \tau^{+} \tau^{-}$ & $s \bar{s} s \bar{s}$ & $s \bar{s} b \bar{b}^{*}$ & $b \bar{b} b \bar{b}$ \\
\hline$\sigma(+-)$ & $4.7 \times 10^{9}$ & 2169 & 232 & 532048 & 3409 & 313 \\
\hline$\sigma(++)$ & $4.7 \times 10^{9}$ & 2288 & 233 & 551620 & 3485 & 317 \\
\hline \multirow[t]{2}{*}{$\sigma(00)$} & $4.7 \times 10^{9}$ & 2228 & 232 & 541824 & 3447 & 315 \\
\hline & \multicolumn{6}{|c|}{$\sqrt{s_{e^{+} e^{-}}}=500 \mathrm{GeV}$} \\
\hline$\sigma(+-)$ & $1.9 \times 10^{9}$ & 1054 & 114 & 241093 & 1629 & 161 \\
\hline$\sigma(++)$ & $1.9 \times 10^{9}$ & 1042 & 115 & 240166 & 1707 & 162 \\
\hline \multirow[t]{2}{*}{$\sigma(00)$} & $1.9 \times 10^{9}$ & 1048 & 114 & 240612 & 1668 & 161 \\
\hline & \multicolumn{6}{|c|}{$\sqrt{s_{e^{+} e^{-}}}=1 \mathrm{TeV}$} \\
\hline
\end{tabular}

*Other c.c.'s are free from SM background. 
TABLE V. Cross sections in femtobarns for the four-fermion final states discussed in the text, for both signals (first three rows for MSSM set A, B, and C, respectively) and backgrounds (last row), for unpolarized beams at $\sqrt{s_{e^{+} e^{-}}}=500 \mathrm{GeV}$, after the cuts in Eq. (10) plus the additional constraint $\left|M_{f f}-M_{\tilde{\nu}}\right|<5 \mathrm{GeV}$, for several sneutrino masses. (Here, $f f$ refers to either lepton-lepton or jet-jet pairs and only one $M_{\tilde{\nu}}$ is required to be reconstructed.) Notice that, for the backgrounds, no summation over $u, d$, and $s$ (light) flavors has been performed in the case of signatures involving $s$ quarks.

\begin{tabular}{ccccccc}
\hline \hline$M_{\tilde{\nu}}(\mathrm{GeV})$ & $e^{+} e^{-} e^{+} e^{-}$ & $\mu^{+} \mu^{-} \tau^{+} \tau^{-*}$ & $\tau^{+} \tau^{-} \tau^{+} \tau^{-}$ & $s \bar{s} \bar{s}$ & $s \bar{s} b \bar{b}^{*}$ & $b \bar{b} b \bar{b}$ \\
\hline 100 & 0.1908 & 0.4650 & 0.2565 & 2.387 & 2.100 & 1.3091 \\
& 1.1865 & 1.4177 & 1.3095 & 2.4466 & 2.1270 & 1.3529 \\
& 1.1865 & 1.4177 & 1.3095 & 2.4466 & 2.1270 & 1.3529 \\
& 382042836 & 56 & 19 & 93366 & 627 & 63 \\
200 & 0.00234 & 0.00695 & 0.00313 & 0.3870 & 0.4019 & 0.17967 \\
& 0.03256 & 0.08342 & 0.04208 & 0.5939 & 0.51151 & 0.30668 \\
& 0.6004 & 0.13705 & 0.07569 & 0.6055 & 0.51651 & 0.31462 \\
& 93921298 & 6.4 & 4.4 & 22248 & 170 & 18 \\
& 0.000171 & 0.000493 & 0.000222 & 0.04387 & 0.0477 & 0.01649 \\
& 0.00112 & 0.003163 & 0.001461 & 0.09135 & 0.07628 & 0.03892 \\
& 0.00267 & 0.007204 & 0.003431 & 0.10293 & 0.08134 & 0.04533 \\
& 19052146 & 3.6 & 1.7 & 3696 & 30 & 3.3 \\
\hline \hline
\end{tabular}

*Other c.c.'s are free from SM background.

thousand events in each of the three channels $\mu^{+} \mu^{-} \tau^{+} \tau^{-}$, $\tau^{+} \tau^{-} \tau^{+} \tau^{-}$, and $b \bar{b} b \bar{b}$, depending on the actual sneutrino mass and assuming a luminosity of $1 \mathrm{ab}^{-1}$.

Furthermore, since typical SM backgrounds have been seen to be less sensitive than the signals to the polarization state of the incoming particles, one may exploit the latter in order to improve the discovery potential of RPV signals at future LCs. If a high, but not unrealistic, degree of polariza- tion of both laser photons and leptonic beams can be achieved, this can be exploited to push the discovery reach in sneutrino mass somewhat beyond the mentioned $M_{\tilde{v}}$ values in the $\tau^{+} \tau^{-} \tau^{+} \tau^{-}$and $b \bar{b} b \bar{b}$ final states (i.e., those most massive in the leptonic and hadronic case, respectively) at $500 \mathrm{GeV}$. In fact, at this energy, the polarization combination in which the electron and positron helicities have the opposite sign and are also opposite to those of the corresponding

TABLE VI. Same as Table V for $\sqrt{s}_{e^{+} e^{-}}=1 \mathrm{TeV}$.

\begin{tabular}{|c|c|c|c|c|c|c|}
\hline$M_{\tilde{\nu}}(\mathrm{GeV})$ & $e^{+} e^{-} e^{+} e^{-}$ & $\mu^{+} \mu^{-} \tau^{+} \tau^{-*}$ & $\tau^{+} \tau^{-} \tau^{+} \tau^{-}$ & $s \bar{s} s \bar{s}$ & $s \bar{s} b \bar{b}^{*}$ & $b \bar{b} b \bar{b}$ \\
\hline \multirow[t]{4}{*}{100} & 0.1313 & 0.3268 & 0.1860 & 1.7951 & 1.6455 & 1.0897 \\
\hline & 0.8168 & 0.9963 & 0.9496 & 1.8394 & 1.6659 & 1.1262 \\
\hline & 0.8168 & 0.9963 & 0.9496 & 1.8394 & 1.6659 & 1.1262 \\
\hline & 206634870 & 48 & 14 & 39993 & 271 & 28 \\
\hline \multirow[t]{4}{*}{200} & 0.00269 & 0.00801 & 0.00401 & 0.4869 & 0.5385 & 0.2621 \\
\hline & 0.03740 & 0.09613 & 0.05383 & 0.7471 & 0.6854 & 0.4474 \\
\hline & 0.06897 & 0.1579 & 0.09681 & 0.7617 & 0.6921 & 0.4590 \\
\hline & 59351446 & 8.0 & 8.2 & 15451 & 163 & 16 \\
\hline \multirow[t]{4}{*}{300} & 0.000486 & 0.001532 & 0.000701 & 0.1459 & 0.1913 & 0.07331 \\
\hline & 0.003192 & 0.009818 & 0.004616 & 0.3039 & 0.3059 & 0.1729 \\
\hline & 0.007567 & 0.02235 & 0.01083 & 0.3424 & 0.3262 & 0.2014 \\
\hline & 23373961 & 3.7 & 1.1 & 7005 & 47 & 7.3 \\
\hline \multirow[t]{4}{*}{400} & 0.000189 & 0.0005867 & 0.000282 & 0.06167 & 0.08462 & 0.03029 \\
\hline & 0.000997 & 0.003023 & 0.001452 & 0.1351 & 0.1432 & 0.07438 \\
\hline & 0.002332 & 0.006881 & 0.003376 & 0.1610 & 0.1579 & 0.09261 \\
\hline & 11211595 & 2.3 & 0.93 & 2393 & 19 & 2.3 \\
\hline \multirow[t]{4}{*}{500} & $8.476 \times 10^{-5}$ & 0.0002556 & 0.000117 & 0.02832 & 0.03792 & 0.01336 \\
\hline & 0.000226 & 0.000685 & 0.000320 & 0.05041 & 0.05710 & 0.02544 \\
\hline & 0.000920 & 0.002706 & 0.001305 & 0.07713 & 0.07362 & 0.04249 \\
\hline & 5294773 & 0.38 & 0.22 & 1335 & 8 & 1.0 \\
\hline
\end{tabular}

*Other c.c.'s are free from SM background. 
TABLE VII. Significances $S / \sqrt{B}$ at $\sqrt{s_{e^{+} e^{-}}}=500 \mathrm{GeV}$ after $1 \mathrm{ab}^{-1}$ for the four-fermion final states discussed in the text, for both signals (first three rows for MSSM set A, B, and C, respectively) and backgrounds (last row), for unpolarized beams, after the cuts in Eq. (10) plus the additional constraint $\left|M_{f f}-M_{\tilde{\nu}}\right|<5 \mathrm{GeV}$, for several sneutrino masses. (Here, $f f$ refers to either lepton-lepton or jet-jet pairs and only one $M_{\tilde{\nu}}$ is required to be reconstructed.) Notice that, for the backgrounds, no summation over $u, d$, and $s$ (light) flavors has been performed in the case of signatures involving $s$ quarks.

\begin{tabular}{lcccccc}
\hline \hline$M_{\tilde{\nu}}(\mathrm{GeV})$ & $e^{+} e^{-} e^{+} e^{-}$ & $\mu^{+} \mu^{-} \tau^{+} \tau^{-}$ & $\tau^{+} \tau^{-} \tau^{+} \tau^{-}$ & $s \bar{s} s \bar{s}$ & $s \bar{s} b \bar{b}$ & $b \bar{b} b \bar{b}$ \\
\hline 100 & 0.00031 & 2.0 & 1.9 & 0.25 & 2.7 & 5.2 \\
& 0.0019 & 6.0 & 9.5 & 0.25 & 2.7 & 5.4 \\
& 0.0019 & 6.0 & 9.5 & 0.25 & 2.7 & 5.4 \\
200 & $7.6 \times 10^{-6}$ & 0.087 & 0.047 & 0.082 & 0.97 & 1.3 \\
& 0.00011 & 1.0 & 0.63 & 0.13 & 1.2 & 2.3 \\
300 & 0.0020 & 1.7 & 1.14 & 0.13 & 1.3 & 2.3 \\
& $1.2 \times 10^{-6}$ & 0.0082 & 0.0054 & 0.023 & 0.28 & 0.29 \\
& $8.1 \times 10^{-6}$ & 0.053 & 0.035 & 0.048 & 0.44 & 0.68 \\
& $1.9 \times 10^{-5}$ & 0.12 & 0.083 & 0.054 & 0.47 & 0.79 \\
\hline \hline
\end{tabular}

laser photons, i.e., $(+-)$, yields, for sneutrino masses in the 100-250 GeV region, signal rates somewhat higher than those induced in the other cases (including that of unpolarised beams), up to a factor of 2. In contrast, for the $\mu^{+} \mu^{-} \tau^{+} \tau^{-}$final state, it is the unpolarized configuration, i.e., (00), that is the most suitable for sneutrino searches in the above mass range. Once the collider energy is raised to 1 $\mathrm{TeV}$, differences between the three polarization combinations almost disappear if $M_{\tilde{\nu}} \lesssim 250 \mathrm{GeV}$. The polarization state in which the electron and positron helicities have the same sign and opposite to the one of the laser photons, i.e., $(++)$, would turn out extremely useful for heavy sneutrino masses, say, when $M_{\tilde{\nu}} \gtrsim \sqrt{s_{e^{+} e^{-}}} / 2$, as here signal rates are consistently and significantly above those induced by the other polarizations, up to a factor of 4 in some instances. Unfortunately, this mass interval is unattainable through present LC designs [DESY TeV Energy Superconducting Linear Accelerator (TESLA), Next Linear Collider (NLC), and Japan Lin- ear Collider (JLC)] and will have to attend for higher collider energies, such as those foreseen for CERN Linear Collider

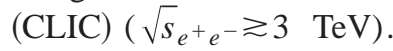

Before closing, two final considerations are in order. First, recall that, as a bonus of the production process considered here, some leptonic signatures which are flavor changing, such as $\mu^{+} \mu^{+} \tau^{-} \tau^{-}$and $\mu^{-} \mu^{-} \tau^{+} \tau^{+}$, would come practically free from SM background, hence promptly detectable at a future LC. Second, that we have not included the effect of finite experimental efficiency in tagging leptons and jets, so that our final significances may be somewhat overestimated. The comment particularly applies to the $4 b$ final state, for which we have assumed throughout a $100 \%$ efficiency for a quadruple $b$ tagging. If one adopts a more realistic $70 \%$ per $b$-jet, significances in the last columns in Tables VII and VIII would go down by a factor of 2, hampering seriously the scope of the hadronic channel. However, one may alternatively consider tagging only a subset of the

TABLE VIII. Same as Table VII for $\sqrt{s}_{e^{+} e^{-}}=1 \mathrm{TeV}$.

\begin{tabular}{ccccccc}
\hline \hline$M_{\tilde{\nu}}(\mathrm{GeV})$ & $e^{+} e^{-} e^{+} e^{-}$ & $\mu^{+} \mu^{-} \tau^{+} \tau^{-}$ & $\tau^{+} \tau^{-} \tau^{+} \tau^{-}$ & $s \bar{s} \bar{s}$ & $s \bar{s} b \bar{b}$ & $b \bar{b} b \bar{b}$ \\
\hline 100 & 0.00029 & 1.5 & 1.6 & 0.28 & 3.1 & 6.5 \\
& 0.0018 & 4.5 & 8.0 & 0.29 & 3.2 & 6.7 \\
& 0.0018 & 4.5 & 8.0 & 0.29 & 3.2 & 6.7 \\
200 & $1.1 \times 10^{-5}$ & 0.090 & 0.044 & 0.12 & 1.3 & 2.1 \\
& 0.00015 & 1.1 & 0.59 & 0.19 & 1.7 & 3.5 \\
& 0.00028 & 1.8 & 1.1 & 0.19 & 1.7 & 3.6 \\
300 & $3.2 \times 10^{-6}$ & 0.025 & 0.021 & 0.055 & 0.88 & 0.86 \\
& $2.1 \times 10^{-5}$ & 0.16 & 0.14 & 0.11 & 1.4 & 2.0 \\
& $4.9 \times 10^{-5}$ & 0.37 & 0.33 & 0.13 & 1.5 & 2.4 \\
400 & $1.8 \times 10^{-6}$ & 0.012 & 0.0092 & 0.039 & 0.61 & 0.63 \\
& $9.4 \times 10^{-6}$ & 0.063 & 0.048 & 0.087 & 1.04 & 1.6 \\
& $2.2 \times 10^{-5}$ & 0.14 & 0.11 & 0.10 & 1.1 & 1.9 \\
500 & $1.2 \times 10^{-6}$ & 0.013 & 0.0079 & 0.025 & 0.42 & 0.42 \\
& $3.1 \times 10^{-6}$ & 0.035 & 0.022 & 0.044 & 0.64 & 0.80 \\
& $1.3 \times 10^{-5}$ & 0.14 & 0.088 & 0.067 & 0.82 & 1.3 \\
\hline \hline
\end{tabular}


TABLE IX. Sneutrino decay width (in $\mathrm{GeV}$ ) in the RPV decay channels relevant to our analysis for the MSSM parameter sets A (top), B (middle), and C (bottom).

\begin{tabular}{llllccc}
\hline \hline$M_{\tilde{\nu}}(\mathrm{GeV})$ & $e^{+} e^{-}$ & $\mu^{+} \tau^{-}$ & $\tau^{+} \tau^{-}$ & $s \bar{s}$ & $s \bar{b}$ & $b \bar{b}$ \\
\hline 100 & 0.0238 & 0.0297 & 0.0248 & 0.8269 & 1.6270 & 0.6169 \\
200 & 0.6474 & 0.6593 & 0.6495 & 2.2536 & 3.8640 & 1.8447 \\
400 & 4.1109 & 4.1345 & 4.1151 & 7.3232 & 10.5492 & 6.5109 \\
100 & 0.0038 & 0.0097 & 0.0049 & 0.8069 & 1.6070 & 0.5970 \\
200 & 0.0642 & 0.0760 & 0.0663 & 1.6704 & 3.2808 & 1.2614 \\
400 & 0.8613 & 0.8850 & 0.8655 & 4.0737 & 7.2996 & 3.2613 \\
100 & 0.0038 & 0.0097 & 0.0049 & 0.8069 & 1.6070 & 0.5970 \\
200 & 0.0294 & 0.0413 & 0.0315 & 1.6356 & 3.2460 & 1.2267 \\
400 & 0.3975 & 0.4212 & 0.4017 & 3.6099 & 6.8359 & 2.7976 \\
\hline \hline
\end{tabular}

four $b$ quarks. The correct estimate of the potential in this channel clearly depends on the tagging strategy of $b$ quarks and can only be obtained in the context of a fully hadronic environment and in the presence of detector effects, which were lacking in our study. However, in the worse case scenario in which the $b \bar{b} b \bar{b}$ signature of RPV sneutrinos produced in the associated mode is swamped by the backgrounds, one should still be able to resort to $\mu^{+} \mu^{-} \tau^{+} \tau^{-}$and $\tau^{+} \tau^{-} \tau^{+} \tau^{-}$. Altogether, we consider the subject raised in this paper of relevance for the physics of future LCs and look forward to experimental studies in the context of the current LC workshops.

\section{ACKNOWLEDGMENTS}

We thank Abdesselam Arhrib for discussions. The work of D.K.G. is supported by the National Science Council of Taiwan under the grant NSC 90-2811-M-002-054 and from the Ministry of Education Academic Excellence Project 89-NFA01-1-4-3 of Taiwan.

\section{APPENDIX}

As intimated in the text, we reproduce here the sneutrino partial widths in the two-body decay channels considered in the paper, see Table IX, for the MSSM parameter sets given in Table II.
[1] ACFA Linear Collider Working Group, K. Abe et al., hep-ph/0109166 and references therein; American Linear Collider Working Group, T. Abe et al., hep-ex/0106055; hep-ex/0106056; hep-ex/0106057; hep-ex/0106058 and references therein; ECFA/DESY LC Physics Working Group, J. A. Aguilar-Saavedra et al., SLAC-REPRINT-2001-002, DESY01-011, DESY-2001-011, DESY-01-011C, DESY-2001-011C, DESY-TESLA-2001-23, DESY-TESLA-FEL-2001-05, ECFA2001-209, K. Abe et al., 2001, hep-ph/0106315; CLIC Study Team, edited by G. Guignard, CERN-2000-008.

[2] I. Ginzburg, G. Kotkin, V. Serbo, and V. Telnov, Nucl. Instrum. Methods Phys. Res. 205, 47 (1983); Nucl. Instrum. Methods Phys. Res. A 219, 5 (1984); V. Telnov, ibid. 294, 72 (1990).

[3] V. Telnov, Nucl. Instrum. Methods Phys. Res. A 472, 43 (2001), and references therein.

[4] F. Cuypers, G.J. van Oldenborgh, and R. Ruckl, Nucl. Phys. B409, 144 (1993).

[5] S. Berge, M. Klasen, and Y. Umeda, Phys. Rev. D 63, 035003 (2001); A. Datta and D. Choudhury, Nucl. Phys. B592, 35 (2001); T. Mayer and H. Fraas, Nucl. Instrum. Methods Phys. Res. A 472, 165 (2001); D. Gorbunov, V. Ilyin, and V. Telnov, ibid. 472, 171 (2001); D.M. Asner, J.B. Gronberg, and J.F. Gunion, hep-ph/0110320, and references therein.

[6] D.E. Groom et al., Particle Data Group, Eur. Phys. J. C 1, 15 (2000).

[7] P. Fayet, Phys. Lett. 69B, 489 (1977); G. Farrar and P. Fayet, ibid. 76B, 575 (1978); N. Sakai and T. Yanagida, Nucl. Phys. B197, 533 (1982); C. Aulakh and R. Mohapatra, Phys. Lett. 119B, 136 (1983).

[8] S. Weinberg, Phys. Rev. D 26, 287 (1982).

[9] L.J. Hall and M. Suzuki, Nucl. Phys. B231, 419 (1984); R. Barbieri and A. Masiero, ibid. B267, 679 (1986); S. Dimopoulos and L.J. Hall, Phys. Lett. B 207, 210 (1988); V. Barger, G.F. Giudice, and T. Han, Phys. Rev. D 40, 2987 (1989); M. Czakon and J. Gluza, hep-ph/0003228; R. Godbole, P. Roy, and X. Tata, Nucl. Phys. B401, 67 (1993); G. Bhattacharyya, J.R. Ellis, and K. Sridhar, Mod. Phys. Lett. A 10, 1583 (1995); G. Bhattacharyya, D. Choudhury, and K. Sridhar, Phys. Lett. B 355, 193 (1995); D. Choudhury and S. Raychaudhury, ibid. 401, 54 (1997); D.K. Ghosh, S. Raychaudhuri, and K. Sridhar, ibid. 396, 177 (1997); K. Huitu, J. Maalampi, M. Raidal, and A. Santamaria, ibid. 430, 355 (1998); G. Moreau, Ph.D. thesis, Paris University, 2000, hep-ph/0012156, and references therein.

[10] Dimopoulos and Hall, [9]; Czakon and Gluza, [9].

[11] Barger, Giudice, and Han, [9].

[12] S. Bar-Shalom, G. Eilam, J. Wudka, and A. Soni, Phys. Rev. D 59, 035010 (1999).

[13] M. Chaichian, K. Huitu, S. Roy, and Z.H. Yu, Phys. Lett. B 518, 261 (2001).

[14] D. K. Ghosh and S. Moretti (in preparation).

[15] K. Jacobs, report UMZ-01-0720, 2001, hep-ex/0107084; see 
also: http://sl.web.cern. ch/SL/opnews/pageswww/lcnov2000/ sld005.htm.

[16] ALEPH Collaboration, D. Abbaneo et al., report CERN-EP/ 2001-094, 2002, hep-ex/0201013.

[17] H. Murayama, I. Watanabe, and K. Hagiwara, HELAS: HELicity Amplitude Subroutines for Feynman Diagram Evaluations, KEK Report No. 91-11, 1992.

[18] T. Stelzer and W.F. Long, Comput. Phys. Commun. 81, 357 (1994).

[19] G.P. Lepage, J. Comput. Phys. 27, 192 (1978).

[20] Hall and Suzuki, Ref. [9]; I-H. Lee, Phys. Lett. 138B, 121 (1984); Nucl. Phys. B246, 120 (1984); S. Dawson, ibid. B261,
297 (1985); F. de Campos, M.A. Garcia-Jareno, A.S. Joshipura, J. Rosiek, and J.W.F. Valle, ibid. B451, 3 (1995); M. Nowakowski and A. Pilaftsis, ibid. B461, 19 (1996); R. Hempfling, ibid. B478, 3 (1996); S. Roy and B. Mukhopadhyaya, Phys. Rev. D 55, 7020 (1997); M. Hirsch, M.A. Díaz, W. Porod, J.C. Romão, and J.W.F. Valle, ibid. 62, 113008 (2000).

[21] Berge, Klasen, and Umeda, in Ref. [5].

[22] See, e.g., B.C. Allanach, A. Dedes, and H.K. Dreiner, Phys. Rev. D 60, 075014 (1999), and references therein.

[23] F. Ledroit and G. Sajot, note GDR-S-008 (ISN, Grenoble, 1998), see: http://qcd.th.u-psud.fr/GDR_SUSY/ GDR_SUSY_PUBLIC/entete_note_publique. 